ARTICLE

DOI: $10.1038 / s 41467-017-01950-1$

\title{
Opposite effects of Activin type 2 receptor ligands on cardiomyocyte proliferation during development and repair
}

\author{
Deepika Dogra', Suchit Ahuja', Hyun-Taek Kim¹, S. Javad Rasouli', Didier Y.R. Stainier ${ }^{1,2}$ \& Sven Reischauer ${ }^{1,2}$
}

Zebrafish regenerate damaged myocardial tissue very effectively. Hence, insights into the molecular networks underlying zebrafish heart regeneration might help develop alternative strategies to restore human cardiac performance. While TGF- $\beta$ signaling has been implicated in zebrafish cardiac regeneration, the role of its individual ligands remains unclear. Here, we report the opposing expression response during zebrafish heart regeneration of two genes, mstnb and inhbaa, which encode TGF- $\beta$ family ligands. Using gain-of-function (GOF) and lossof-function (LOF) approaches, we show that these ligands mediate inverse effects on cardiac regeneration and specifically on cardiomyocyte (CM) proliferation. Notably, we find that Inhbaa functions as a CM mitogen and that its overexpression leads to accelerated cardiac recovery and scar clearance after injury. In contrast, mstnb GOF and inhbaa LOF both lead to unresolved scarring after cardiac injury. We further show that Mstnb and Inhbaa inversely control Smad2 and Smad3 transcription factor activities through alternate Activin type 2 receptors.

\footnotetext{
${ }^{1}$ Department of Developmental Genetics, Max Planck Institute for Heart and Lung Research, 61231 Bad Nauheim, Germany. ${ }^{2}$ Department of Biochemistry and Biophysics, University of California San Francisco, San Francisco, 94158 CA, USA. Correspondence and requests for materials should be addressed to S.R. (email: sven.reischauer@mpi-bn.mpg.de)
} 
T he adult mammalian heart is incompetent to regenerate damaged muscle tissue post myocardial infarction (MI). Instead, lost myocardium is replaced by a functionally and electrically inert fibrotic scar, resulting in compromised cardiac performance and arrhythmia ${ }^{1}$. As a consequence, $\mathrm{MI}$ is a leading cause of death and morbidity worldwide ${ }^{2}$. Several active fields of research are trying to address this problem by developing various regenerative approaches focusing on the engraftment of stem cellderived CMs into injured hearts ${ }^{3}$, the stimulation of CM proliferation in situ $^{4}$, or the in situ trans-differentiation of fibroblasts into functional $\mathrm{CMs}^{5}$.
In contrast to mammals, several other vertebrates, including zebrafish, can regenerate injured or lost myocardial tissue ${ }^{6}$ after multiple types of injury, including ventricular resection ${ }^{6}$, cryoinjury $^{7}$, or genetic CM ablation ${ }^{8}$. Consequently, zebrafish serve as an established model to investigate the process of cardiac regeneration. Lineage tracing experiments have reported that remaining CMs in the vicinity of the injured area undergo dedifferentiation and proliferation to give rise to new CMs that subsequently integrate and functionally couple to the remaining myocardium 9 , 10. Hence, an in-depth understanding of the cellular and molecular processes controlling cardiac
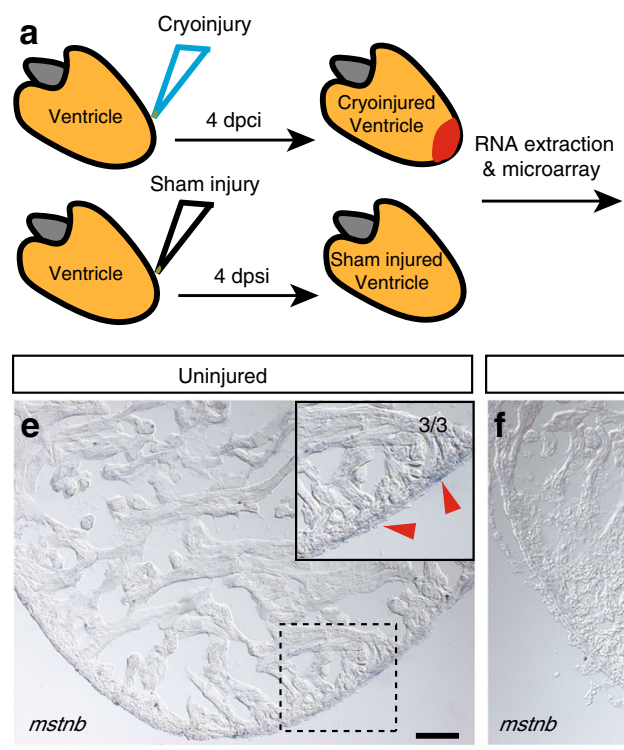

b Gene expression analysis
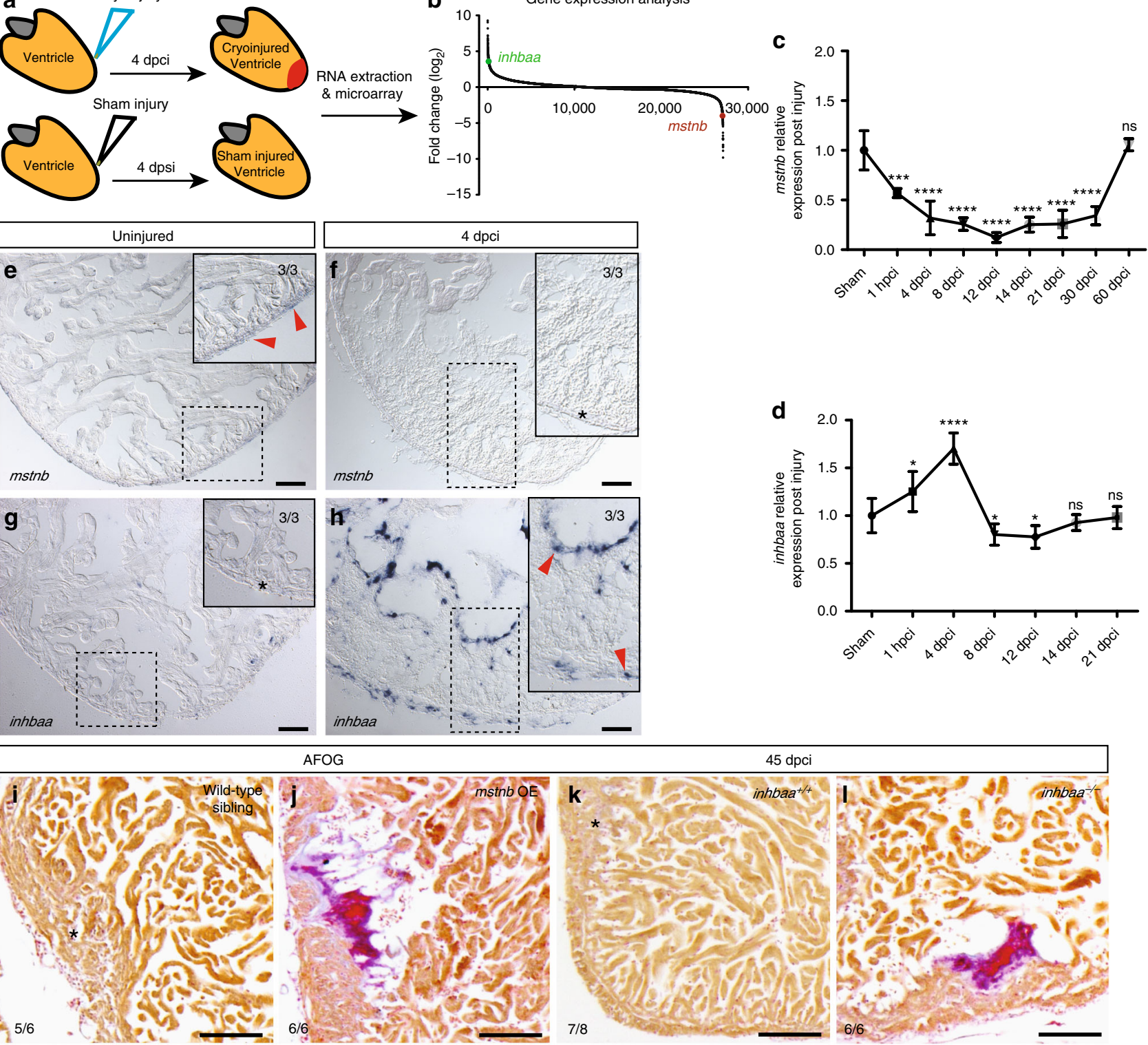

Fig. 1 mstnb and inhbaa have opposing response and functions during zebrafish cardiac regeneration. a Schematic representation of injury and sample preparation for microarray analysis $(n=12$ hearts). b Averaged transcriptional gene expression changes post cryoinjury as assessed by microarray analysis (inhbaa and mstnb indicated). c, d Temporal RT-qPCR analysis for mstnb and inhbaa expression post cryoinjury $(n=2 \times 3$ cardiac ventricles assessed as two biological and two technical replicates for each time point, data are mean \pm s.e.m., ns: no significant changes observed, ${ }^{\star} P \leq 0.05$, ${ }^{\star \star \star} P \leq 0.001$, and ${ }^{\star \star \star \star} P \leq$ $0.0001-S t u d e n t ' s t$ test, two-tailed). e-h In situ hybridization for mstnb and inhbaa expression on uninjured and 4 dpci adult zebrafish heart sections. Higher magnifications of dashed boxes in $\mathbf{e}-\mathbf{h}$ are shown in upper right corners. RNA probe signal is indicated by red arrowheads and the absence of signal is indicated by asterisks. The numerators indicate the number of hearts with a particular pattern of signal, and the denominators the total number of hearts analyzed. i-I AFOG staining of sections from wild-type sibling, mstnb OE, inhbaa ${ }^{+/+}$, and inhbaa ${ }^{-/-}$cryoinjured hearts at 45 dpci. Healthy myocardium in orange, fibrin in red, collagen in blue. Asterisks indicate the regions of resolved scarring. The numerators indicate the number of hearts with a particular pattern of scarring, and the denominators the total number of hearts analyzed. Scale bars: in situ hybridization images, $50 \mu m$; AFOG staining images, 100 $\mu \mathrm{m}$ 
regeneration appears instrumental to develop alternative therapeutic strategies.

Various signaling pathways including epidermal growth factor (EGF), bone morphogenetic protein, vascular endothelial growth factor, interleukin 6 class cytokines and others have been implicated in the process of cardiac regeneration ${ }^{11-13}$. Importantly, only Neuregulins (Nrg) and their co-receptor ERBB2 have so far been reported to possess mitogenic activity on CMs, not only after injury, but also on the healthy myocardium of fish and mice $e^{4,14-}$ 17. Consequently, Nrg is subject to ongoing research to evaluate its therapeutic potential ${ }^{18}$. Members of the transforming growth factor beta (TGF- $\beta$ ) signaling pathway have been implicated in various developmental ${ }^{19}$ and disease conditions ${ }^{20}$, but the role of its numerous components in cardiac regeneration is poorly understood. The vertebrate TGF- $\beta$ /Activin subfamily (TGF- $\beta$ family) of ligands is comprised of Activins (INHBA, INHBB), GDFs (Myostatin/GDF8, GDF11) and TGF- $\beta$ (TGFB1, TGFB2, and TGFB3) which bind Activin type 2 receptors (ACVR2A, ACVR2B, TGFBR2), leading to the recruitment and activation of Activin type 1 receptors (ACVR1B, TGFBR1, ACVR1C). Canonically, this process is followed by phosphorylation of the signal transducers Smad2/3, which bind to Smad4 and translocate to the nucleus, thereby modulating the expression of target genes ${ }^{21-23}$. Several non-canonical TGF- $\beta$ pathways have also been reported, which involve mitogen-activated protein kinases (MAPK) and phosphatidylinositol-3-kinase/ $\mathrm{Akt}^{24}$. In the diseased mammalian heart, enhanced TGF- $\beta$ signaling through upregulation of Myostatin $(\mathrm{MSTN})^{25}$, Inhibin betaA (INHBA) ${ }^{26}$, and TGF- $\beta^{27}$, stimulates hypertrophy, fibrosis, apoptosis, and endothelial-mesenchymal transition ${ }^{28,29}$. Moreover, global myocardial inhibition of TGF- $\beta$ signaling through CM-specific Tgfbr 2 deletion reduces pathological remodeling in response to sustained pressure overload ${ }^{30}$. In zebrafish, TGF- $\beta$ signaling is essential for heart regeneration as chemical inhibition of Activin type 1 receptors suppresses $\mathrm{CM}$ proliferation and compromises overall cardiac regeneration $^{31}$. MSTN, a well-known negative regulator of skeletal muscle growth ${ }^{32,33}$, has been implicated in the development of cardiac hypertrophy in mammals ${ }^{34}$, 35 . Further, the absence of MSTN enhances murine skeletal muscle regeneration $^{36}$, and a recently developed monoclonal antibody against MSTN shows therapeutic potential in the treatment of skeletal muscle atrophy ${ }^{37}$. inhba, also known as activin betaA, promotes wound closure by regulating c-Jun phosphorylation and blastema proliferation during zebrafish fin regeneration ${ }^{38}$. In sum, the role of TGF- $\beta$ signaling and its various ligands during heart regeneration and pathology remains unclear. Using gene expression profiling, we identified the opposing expression response of mstnb and inhbaa to cardiac cryoinjury, calling for a detailed investigation of their specific roles during cardiac regeneration.

Here we show that these two TGF- $\beta$ family ligands antagonize one another during zebrafish cardiac regeneration. While mstnb is robustly and continuously downregulated after cryoinjury in the adult zebrafish heart, inhbaa is upregulated. Loss of mstnb function and activation of inhbaa expression are both beneficial for CM proliferation and lead to enhanced cardiac regeneration. Notably, the overexpression (OE) of inhbaa alone is sufficient to induce CM proliferation independently of the well-known Nrg-ErbB signaling pathway. Furthermore, we show that Mstnb and Inhbaa function through alternate receptor complexes to control the activities of the signal transducers, Smad2 and Smad3, and regulate CM proliferation during development.

\section{Results}

mstnb and inhbaa expression show opposing response to injury. In order to identify genes differentially regulated during adult zebrafish heart regeneration, we performed microarraybased expression profiling of whole hearts 4 days post sham injury (dpsi) vs. 4 days post cryoinjury (dpci) (Fig. 1a). Interestingly, we detected opposing expression response of two genes, mstnb and inhbaa, encoding TGF- $\beta$ family ligands (Fig. $1 \mathrm{~b}$; raw data have been deposited in the NCBI-Gene Expression Omnibus WebsiteGSE89259). To obtain more detailed temporal expression data, we quantified mstnb and inhbaa expression in sham and cryoinjured ventricles at different time points post injury using real-time quantitative PCR (RT-qPCR). Consistent with our microarray data, we observed a rapid reduction of mstnb transcript levels in regenerating ventricles compared to sham injury, as early as $1 \mathrm{~h}$ post cryoinjury (hpci) (Fig. 1c). Interestingly, mstnb expression did not return to basal levels before $60 \mathrm{dpci}$ (Fig. 1c), when regeneration was completed $^{7}$, suggesting that a continuous reduction of mstnb expression is important for cardiac regeneration. On the contrary, inhbaa showed rapid upregulation, peaking at 4 dpci (Fig. 1d); however, by 8 dpci inhbaa expression had returned to sham levels (Fig. 1d), indicating a principal role for inhbaa during the early phase of cardiac regeneration. To complement the temporal expression data, we analyzed the spatiotemporal pattern of mstnb and inhbaa expression by in situ hybridization on sections. Interestingly, mstnb expression was strongest in the ventricular wall of uninjured hearts (Fig. 1e), and it was reduced below detection levels in 4 dpci samples (Fig. 1f). We also quantified mstnb expression levels in uninjured adult hearts by RT-qPCR on laser micro dissected (LMD) tissues and observed higher levels in the ventricular wall over trabecular tissues (Supplementary Fig. 1a). In contrast, no expression of inhbaa could be detected in uninjured hearts (Fig. 1g), while it was prominent proximal to the injury site at $4 \mathrm{dpci}$ (Fig. 1h), consistent with published data based on RNA tomography ${ }^{11}$. To complete our assessment, we examined other TGF- $\beta /$ Activin ligands and their receptors (refer to Supplementary Table 1 for gene names). Even at a later time point ( $6 \mathrm{dpci})$, inhbaa and its paralogs $i n h b b$ and $t g f b 3$ are the only significantly induced TGF- $\beta$ family ligand encoding genes, with inhbaa showing the most robust upregulation (Supplementary Fig. 1b, c). Taken together, the expression patterns of inhbaa and mstnb clearly show different spatiotemporal changes during cardiac regeneration.

mstnb GOF and inhbaa LOF lead to unresolved scarring. Following our observation that the expression of mstnb decreases during cardiac regeneration, we wanted to analyze the effect of sustained mstnb expression in regenerating hearts. Hence, we generated a transgenic zebrafish line for CM-specific constitutive OE of mstnb, Tg(myl7:mstnb-2A-H2B-EGFP) (mstnb OE hereafter) (Supplementary Fig. 1d-f). As assessed by RT-qPCR, mstnb transcript levels are highly increased in our transgenic mstnb $\mathrm{OE}$ line compared to non-transgenic siblings (Supplementary Fig. 1g). The gross morphology of adult mstnb OE fish and the morphology of their hearts however appear unaffected (Supplementary Fig. 1h-k), indicating that cardiac-specific mstnb $\mathrm{OE}$ does not affect the development or growth of the zebrafish heart, unlike in mouse hearts, where its $\mathrm{OE}$ is reported to cause interstitial fibrosis with compromised cardiac output ${ }^{39}$.

Next, to determine the role of inhbaa during cardiac regeneration, we generated a mutant allele using transcription activator-like effector nuclease (TALEN)-induced mutagenesis. A TALEN targeting the TGF- $\beta$ propeptide domain was designed (Supplementary Fig. 1l) and a 17 bp deletion allele, inhbaa ${ }^{\text {nns37 }}$ (Supplementary Fig. $1 \mathrm{~m}$ ), which is predicted to encode a truncated protein (Supplementary Fig. 1n) was recovered. In addition, inhbaa transcript levels were found to be significantly reduced in $\mathrm{inhbaa}^{-/-}$compared to inhbaa ${ }^{+/+}$siblings as shown 

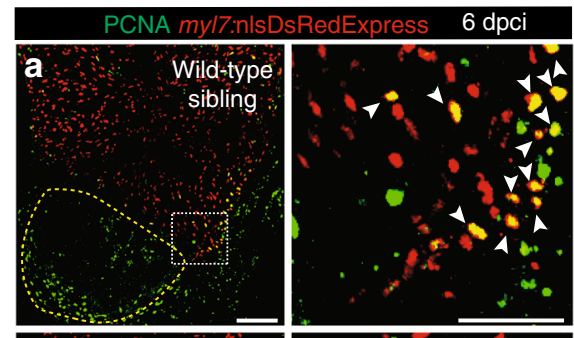

C
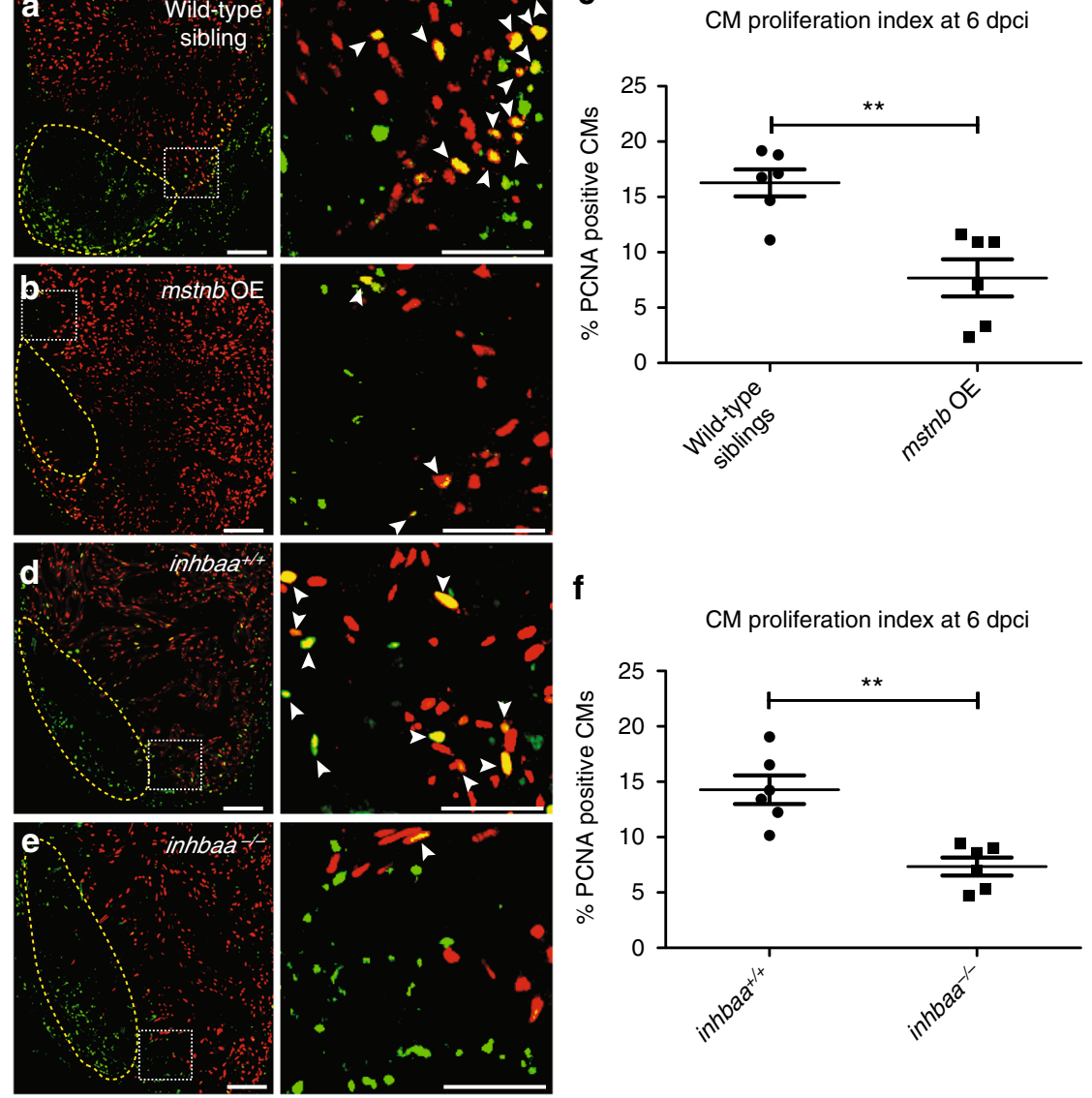

Fig. 2 mstnb GOF and inhbaa LOF suppress CM proliferation during cardiac regeneration. a, b Sections of wild-type sibling and mstnb OE cryoinjured hearts in $T g$ (myl7:nlsDsRedExpress) background at $6 \mathrm{dpci} \alpha$-DsRed (red), PCNA (green). Yellow dotted regions delineate the injured area. Higher magnifications of dashed boxes in $\mathbf{a}, \mathbf{b}$ are shown on right side. White arrowheads point to proliferating CMs (PCNA $\left.{ }^{+} / \operatorname{DsRed}^{+}\right)$. c Quantification of CM proliferation in wildtype sibling $(n=6)$ and mstnb OE $(n=6)$ cryoinjured hearts in the $100 \mu \mathrm{m}$ region adjacent to the injured area at 6 dpci. d, e Sections of inhbaa ${ }^{+/+}$and inhbaa ${ }^{-/-}$cryoinjured hearts in Tg(myl7:nlsDsRedExpress) background at $6 \mathrm{dpci}$; $\alpha$-DsRed (red), PCNA (green). Higher magnifications of dashed boxes in d, e are shown on right side. $\mathbf{f}$ Quantification of CM proliferation in inhbaa ${ }^{+/+}(n=6)$ and inhbaa ${ }^{-/-}(n=6)$ cryoinjured hearts in the $100 \mu \mathrm{m}$ region adjacent to the injured area at $6 \mathrm{dpci}$. All cell counts were performed on three sections from each heart. Each data point on dot plot represents one heart (data are mean \pm s.e.m., ${ }^{\star \star} P \leq 0.01-$ Student's $t$ test, two-tailed). Scale bars: heart sections, $100 \mu \mathrm{m}$; higher magnifications, $50 \mu \mathrm{m}$

by RT-qPCR (Supplementary Fig. 1o), suggesting active mRNA degradation. Similar to mstnb OE animals, inhbaa ${ }^{-/-}$zebrafish do not exhibit any gross morphological defects and their hearts appear indistinguishable from those of wild-type siblings (Supplementary Fig. 1p-s), indicating that inhbaa does not play a critical role during zebrafish development.

The zebrafish heart responds to cardiac injury with the formation of a transient fibrotic scar, which is progressively replaced by newly formed healthy myocardium within two months ${ }^{7}$. To assess any possible defect in the process of cardiac regeneration, we tested for scar resolution in cryoinjured mstnb $\mathrm{OE}$ and $\mathrm{inhbaa}^{-/-}$hearts at $45 \mathrm{dpci}$. By performing acid fuchsin orange G (AFOG) staining on sections, we observed that $45 \mathrm{dpci}$ $m s t n b$ OE and $i n h b a a^{-/-}$hearts were unable to resolve their scar, in contrast to wild-type siblings (Fig. 1i-l) of the same regenerative stage. These data show that both mstnb GOF and inhbaa LOF interfere with cardiac regeneration and consequently cause reduced scar clearance.

mstnb GOF and inhbaa LOF impair CM proliferation post injury. Cardiac regeneration relies on the dedifferentiation and cell cycle re-entry of the spared $\mathrm{CMs}^{9}$. To test whether inhbaa deficiency or mstnb OE modulate CM dedifferentiation, we assessed the expression of embryonic cardiac myosin heavy chain (embCMHC), a marker of dedifferentiated $\mathrm{CMs}^{40}$, at $6 \mathrm{dpci}$. We could not observe any obvious differences in embCMHC expression in mstnb OE or inhbaa ${ }^{-/-}$hearts compared to their wild-type siblings (Supplementary Fig. 2a-d). Next, we tested whether CM proliferation and cell cycle re-entry was affected in mstnb OE and inhbaa ${ }^{-1-}$ fish at 6 dpci. We used $\operatorname{Tg}(m y l 7$ : $n l s D s R e d E x p r e s s)$ fish and performed immunostaining for DsRed and PCNA (cell cycle stage marker), and quantified CM proliferation near the injured area. We observed a $53 \%( \pm 13 \%$ s.e.m.) decrease in CM proliferation in mstnb OE compared to control (Fig. 2a-c), suggesting that mstnb has a significant inhibitory effect on CM proliferation during cardiac regeneration. Similarly, we observed a $49 \%( \pm 11.5 \%$ s.e.m.) reduction in CM proliferation in inhbaa $a^{-/-}$compared to inhbaa ${ }^{+/+}$animals (Fig. 2d-f), indicating that inhbaa is instrumental for CM proliferation in the regenerating heart. Taken together, these results show that mstnb GOF and inhbaa LOF negatively affect CM proliferation, indicating that these two TGF- $\beta$ family ligands have opposite functions during cardiac regeneration.

mstnb LOF and inhbaa GOF promote CM proliferation. Further, as a complementary approach, we examined the effects of 
mstnb LOF and inhbaa GOF on cardiac development and regeneration. In order to analyze the effect of the loss of mstnb, we generated mstnb mutant fish using a TALEN targeting the region after the signal peptide domain (Supplementary Fig. 3a) and recovered a $10 \mathrm{bp}$ deletion allele, $m s t n b^{b n s 5}$ (Supplementary Fig. 3b), which is predicted to encode a truncated protein (Supplementary Fig. 3c). According to our RT-qPCR data, mstnb transcript levels are significantly reduced in $m s t n b^{-/-}$compared to $m s t n b^{+/+}$(Supplementary Fig. 3d), suggesting mRNA decay.
As previously reported in other species ${ }^{32,33}$, adult $m s t n b^{-/-}$fish appear hypermuscular, suggesting a conserved function of Mstn in skeletal muscle growth. Interestingly, we also observed increased heart size in $m s t n b^{-/}$with a thickened ventricular wall (Supplementary Fig. 3e-j, l), the principal expression domain of $m s t n b$ as detailed previously. Notably, the eye size remained unaffected in $m s t n b^{-/}$compared to wild-type siblings (Supplementary Fig. 3k). To better understand whether the increase in ventricular wall thickness was a consequence of increased CM
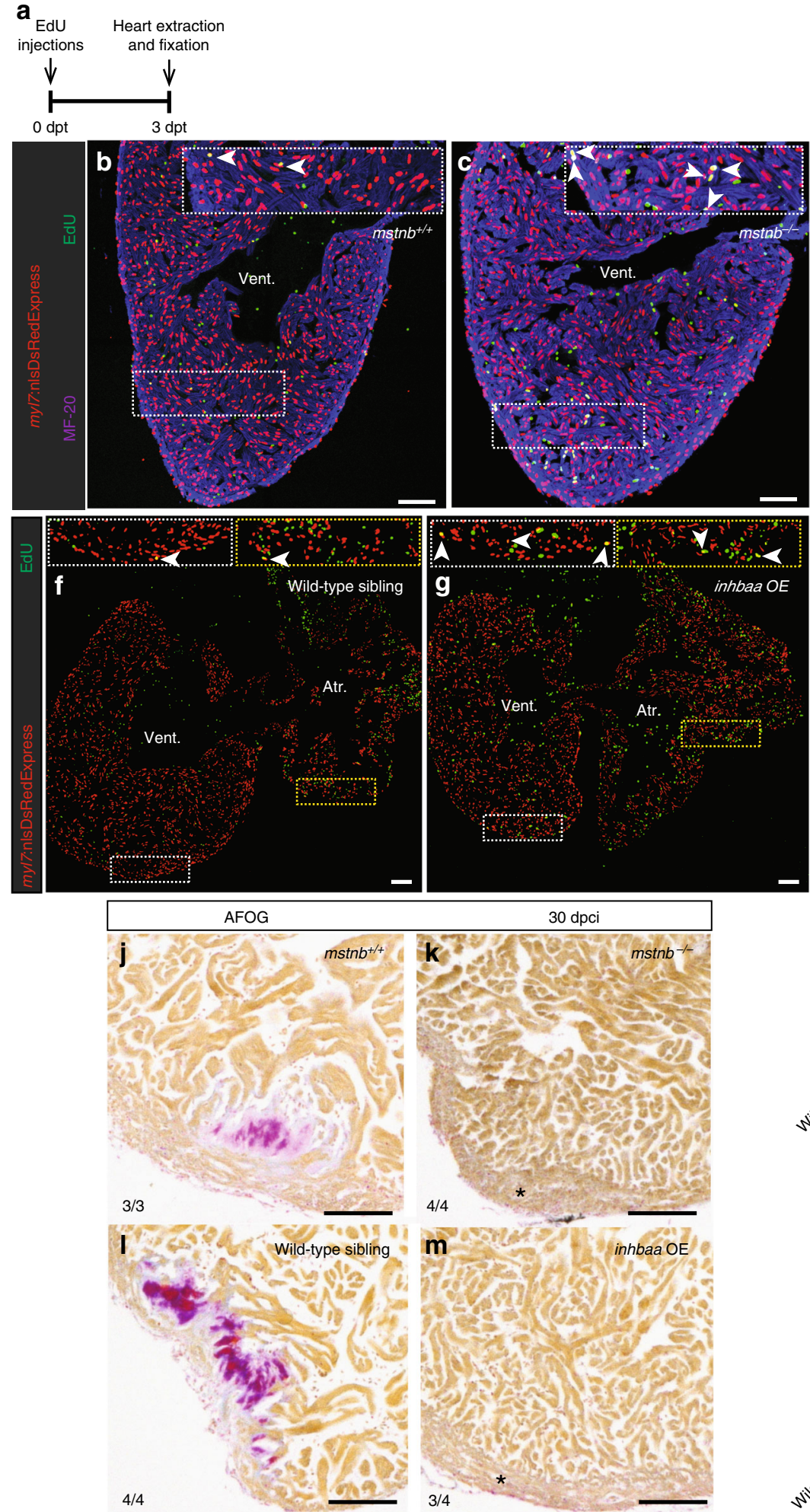
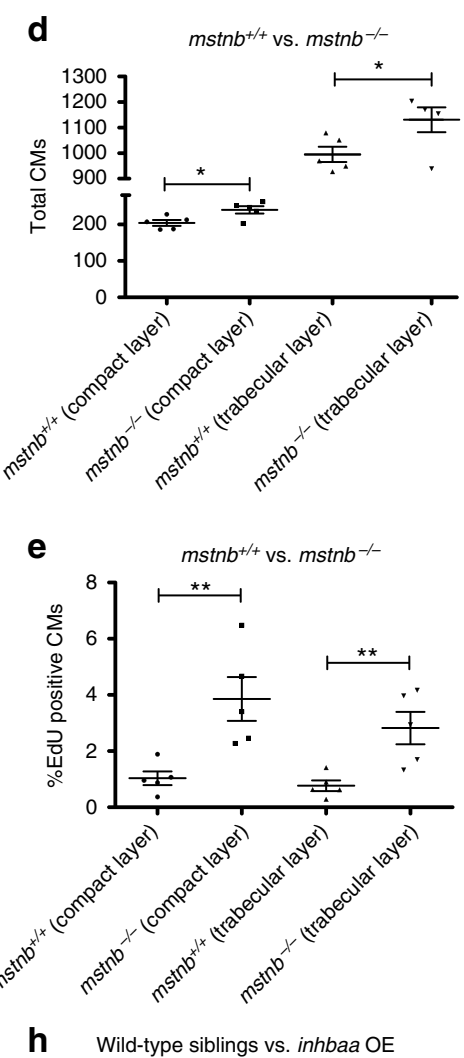

h Wild-type siblings vs. inhbaa OE

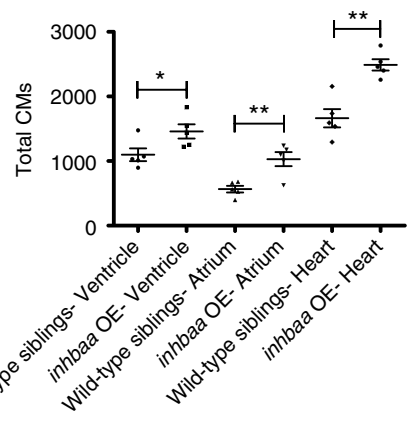

i

Wild-type siblings vs. inhbaa OE

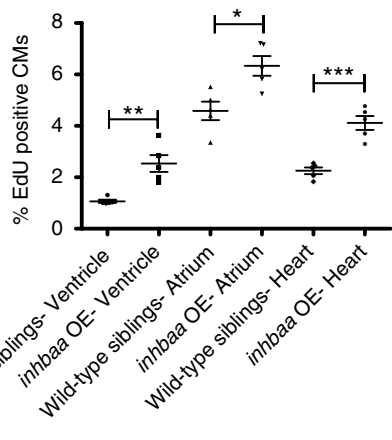


proliferation in the compact layer, we injected EdU in adult $\mathrm{Tg}$ (myl7:nlsDsRedExpress) fish and performed immunostainings for DsRed and CM-specific myosin heavy chain (MF-20), followed by EdU labeling on cardiac sections (Fig. 3a). We quantified the total number of CMs as well as EdU incorporation in CMs in the compact and trabecular layers of the ventricle. Notably, hearts from $m s t n b^{-/}$animals showed a significant increase compared to siblings in the total number of CMs and the number of EdU incorporating $\mathrm{CMs}$, in both the wall and the trabeculae of the ventricle (Fig. $3 \mathrm{~b}-\mathrm{e}$ ). Our results therefore suggest that mstnb LOF promotes CM proliferation, leading to cardiac hyperplasia in zebrafish, unlike in Mstn-knockout mice which respond by cardiac hypertrophy ${ }^{34,35}$.

Next, we generated a transgenic zebrafish line for CM-specific constitutive $\mathrm{OE}$ of inhbaa, Tg(myl7:inhbaa-2A-H2B-EGFP) (inhbaa OE hereafter) (Supplementary Fig. $4 \mathrm{a}-\mathrm{c}$ ), resulting in strongly increased inhbaa transcript levels compared to control (Supplementary Fig. 4d). The majority of adult (3-6 months post fertilization (mpf)) inhbaa OE fish appear morphologically normal when compared to non-transgenic fish; however, the inhbaa OE hearts are significantly enlarged and show dense trabeculation in both chambers, whereas the eye size is unaffected (Supplementary Fig. 4e-k). During late adult stages ( $>6 \mathrm{mpf}$ ), we observed $\sim 20 \%$ of the inhbaa OE fish developing pericardial edema with abnormally enlarged atria, indicating symptoms of a failing heart due to prolonged inhbaa OE. Further, to investigate the causality of cardiac enlargement and hypertrabeculation observed in inhbaa OE hearts of young adults, we performed EdU injections in adult $\mathrm{Tg}$ (myl7:nlsDsRedExpress) fish and immunostained for DsRed, followed by EdU labeling (Fig. 3a). Quantification of total number of CMs as well as EdU incorporation in CMs was performed for both chambers. We detected a significant increase in the total number of CMs, as well as in the number of EdU incorporating CMs in inhbaa OE hearts compared to control (Fig. 3f-i). Further, we tested whether increased CM proliferation helps these hearts after injury. Thus, we compared $m s t n b^{-/-}$and inhbaa OE hearts with their siblings at $30 \mathrm{dpci}$, a time point at which wild-type cryoinjured hearts still retain scarring ${ }^{7}$. By performing AFOG staining on sections, we observed that $30 \mathrm{dpci} m s t n b^{-/-}$and inhbaa OE hearts had completely resolved scars, in contrast to their wild-type siblings (Fig. $3 j-m$ ). Taken together, these results consolidate the finding that mstnb and inhbaa have opposing effects on CM proliferation during regeneration. Intriguingly, inhbaa $\mathrm{OE}$ and mstnb loss-of-function lead to CM hyperplasia, suggesting that inhbaa acts as a mitogen during development and repair.

\section{inhbaa OE promotes CM proliferation independent of ErbB2.} To further investigate the pro-mitogenic effect of inhbaa $\mathrm{OE}$ on CMs, we analyzed CM proliferation in larval hearts at $120 \mathrm{~h}$ post fertilization (hpf). CM labeling was achieved using the $T g$ (myl7: nlsDsRedExpress) background, which again was combined with an EdU incorporation assay, followed by immunostaining for
DsRed (Fig. 4a). We observed an increase of $35 \%( \pm 11 \%$ s.e.m.) in the number of EdU incorporating CMs in inhbaa OE larvae compared to control (Fig. $4 \mathrm{~b}-\mathrm{d}$ ), indicating that inhbaa OE also promotes CM proliferation at early stages. We next wanted to analyze whether the effects of inhbaa $\mathrm{OE}$ on $\mathrm{CM}$ proliferation depended on the $\mathrm{Nrg}-\mathrm{ErbB}$ signaling pathway, a well-known regulator of $\mathrm{CM}$ proliferation ${ }^{9-16}$. Thus, we injected myl7: inhbaa-2A-H2B-EGFP and myl7:H2B-EGFP plasmid DNA in embryos from $e r b b 2^{\text {st61 }}$ heterozygote intercrosses and performed EdU incorporation analysis (Fig. 4e), followed by genotyping. Examining $\mathrm{GFP}^{+} \mathrm{CMs}$ in erbb2 $2^{\text {st61 }}$ homozygous mutants at 120 hpf, we found that a significant amount of $\mathrm{erbb}^{-/-} \mathrm{CMs}$ expressing inhbaa $\mathrm{OE}$ were $\mathrm{EdU}^{+}$while $\mathrm{erbb}^{-/-} \mathrm{CMs}$ expressing GFP alone did not show any signs of EdU incorporation (Fig. 4f-h). Next, we treated inhbaa OE larvae and wild-type siblings with the established ErbB2 inhibitor PD168393 and analyzed CM proliferation (Supplementary Fig. 5a). We observed that pharmacological inhibition of ErbB2 significantly reduced $\mathrm{CM}$ proliferation, as described previously ${ }^{16}$, and interestingly, that inhbaa $\mathrm{OE}$ was able to rescue this effect. We found that inhbaa $\mathrm{OE}$ induces an increase of $77.5 \%( \pm 18 \%$ s.e.m. $)$ in the number of EdU incorporating CMs compared to wild-type siblings under ErbB2-blocking conditions (Supplementary Fig. $5 b-d)$.

As recently reported, in zebrafish, Nrg2a signals through ErbB2 to induce $\mathrm{CM}$ trabeculation and proliferation ${ }^{17}$. We were interested to compare the effects of inhbaa and $n r g 2 a \mathrm{OE}$, and thus decided to test whether the OE of both ligands resulted in an additive effect on $\mathrm{CM}$ proliferation. Thus, by crossing inhbaa $\mathrm{OE}$ and $\mathrm{Tg}$ (myl7:nrg2a-p2a-tdTomato) (nrg2a OE hereafter) fish, we analyzed CM proliferation in $120 \mathrm{hpf} T g(m y l 7: n l s D s R e d E x p r e s s)$ larvae, by performing immunostaining for DsRed, followed by EdU labeling (Fig. 4i) and genotyping. As expected from our before-mentioned observations and recently published work ${ }^{17}$, there was an increase of $38 \%( \pm 7.5 \%$ s.e.m.) and $55 \%( \pm 8 \%$ s.e. $\mathrm{m}$.) in the number of EdU incorporating CMs in inhbaa $\mathrm{OE}$ and $n r g 2 a$ OE larvae, respectively (Fig. $4 \mathrm{j}-1, \mathrm{n}$ ). However, we did not observe any additive effects from overexpressing both ligands, which resulted in a $53 \%$ ( $\pm 6 \%$ s.e.m.) increase in the number of EdU incorporating CMs, similar to $n r g 2 a$ OE alone (Fig. $4 \mathrm{~m}, \mathrm{n}$ ). This result might indicate that $\mathrm{CM}$ proliferation reaches its maximum by $n r g 2 a$ OE alone, as supported by the minimal variability across the assessed samples, or an interference of Inhbaa-mediated proliferation by ErbB signaling. Altogether, these data show that Inhbaa can promote CM proliferation during development and without the need of regenerative stimuli, and that the mitogenic activity of Inhbaa on CMs acts independently of ErbB2 receptor activity.

inhbaa and mstnb compete to regulate CM proliferation. Next, to investigate whether mstnb and inhbaa collaboratively regulate CM proliferation, we crossed our inhbaa OE line with the mstnb OE line in presence of the CM marking $T g(m y l 7: n l s D s R e d E x p r e s s)$

\footnotetext{
Fig. 3 mstnb LOF and inhbaa GOF positively affect physiological CM proliferation and cardiac regeneration. a Experimental setup of EdU treatment, followed by heart extraction and fixation. b, c Sections of $m s t n b^{+/+}$and $m s t n b^{-/-}$adult hearts in $T g$ (myl7:n/sDsRedExpress) background; $\alpha$-DsRed (red), MF-20 (blue), EdU (green). Higher magnifications of dashed boxes in $\mathbf{b}, \mathbf{c}$ are shown in upper right corners. White arrowheads point to $\mathrm{EdU}^{+} / \mathrm{DsRed}^{+} \mathrm{CMs}$. $\mathbf{d}, \mathbf{e}$ Quantification of total CMs (DsRed ${ }^{+}$) and EdU incorporating CMs (EdU $\left.{ }^{+} / \operatorname{DsRed}^{+}\right)$in the compact and trabecular layers of $m s t n b^{+/+}(n=5)$ and $\mathrm{mstnb}^{-/-}$ $(n=5)$ ventricles. $\mathbf{f}, \mathbf{g}$ Sections of wild-type sibling and inhbaa OE adult hearts in Tg(myl7:nlsDsRedExpress) background; $\alpha$-DsRed (red), EdU (green). Higher magnifications of dashed boxes in $\mathbf{f}, \mathbf{g}$ are shown in upper left and upper right corners. $\mathbf{h}$, $\mathbf{i}$ Quantification of total CMs (DsRed ${ }^{+}$) and EdU incorporating $\mathrm{CMs}\left(\mathrm{EdU}^{+} / \mathrm{DsRed}^{+}\right)$in wild-type sibling $(n=5)$ and inhbaa $\mathrm{OE}(n=5)$ hearts. All cell counts were performed on three sections from each heart. Each data point on dot plot represents one heart (data are mean \pm s.e.m., ${ }^{\star} P \leq 0.05$, ${ }^{\star \star} P \leq 0.01,{ }^{\star \star \star} P \leq 0.001-$ Student's $t$ test, two-tailed). $\mathbf{j}-\mathbf{m}$ AFOG staining of sections from mstnb ${ }^{+/+}(n=3), m s t n b^{-/-}(n=4)$, wild-type sibling $(n=4)$, and inhbaa OE $(n=4)$ cryoinjured hearts at 30 dpci. Asterisks indicate the regions of resolved scarring. The numerators indicate the number of hearts with a particular pattern of scarring, and the denominators the total number of hearts analyzed. Scale bars, $100 \mu \mathrm{m}$. dpt, days post treatment; vent., ventricle; atr., atrium
} 
a
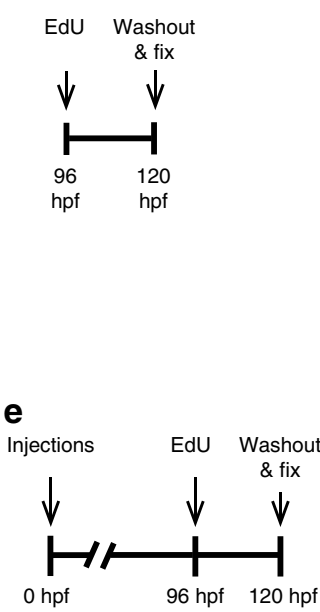

i

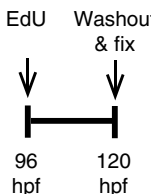

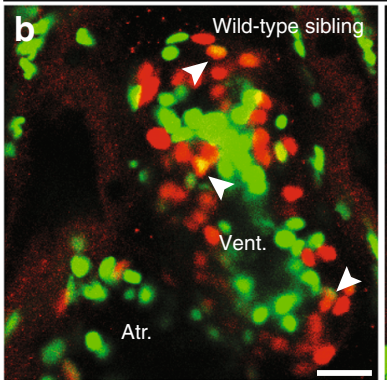
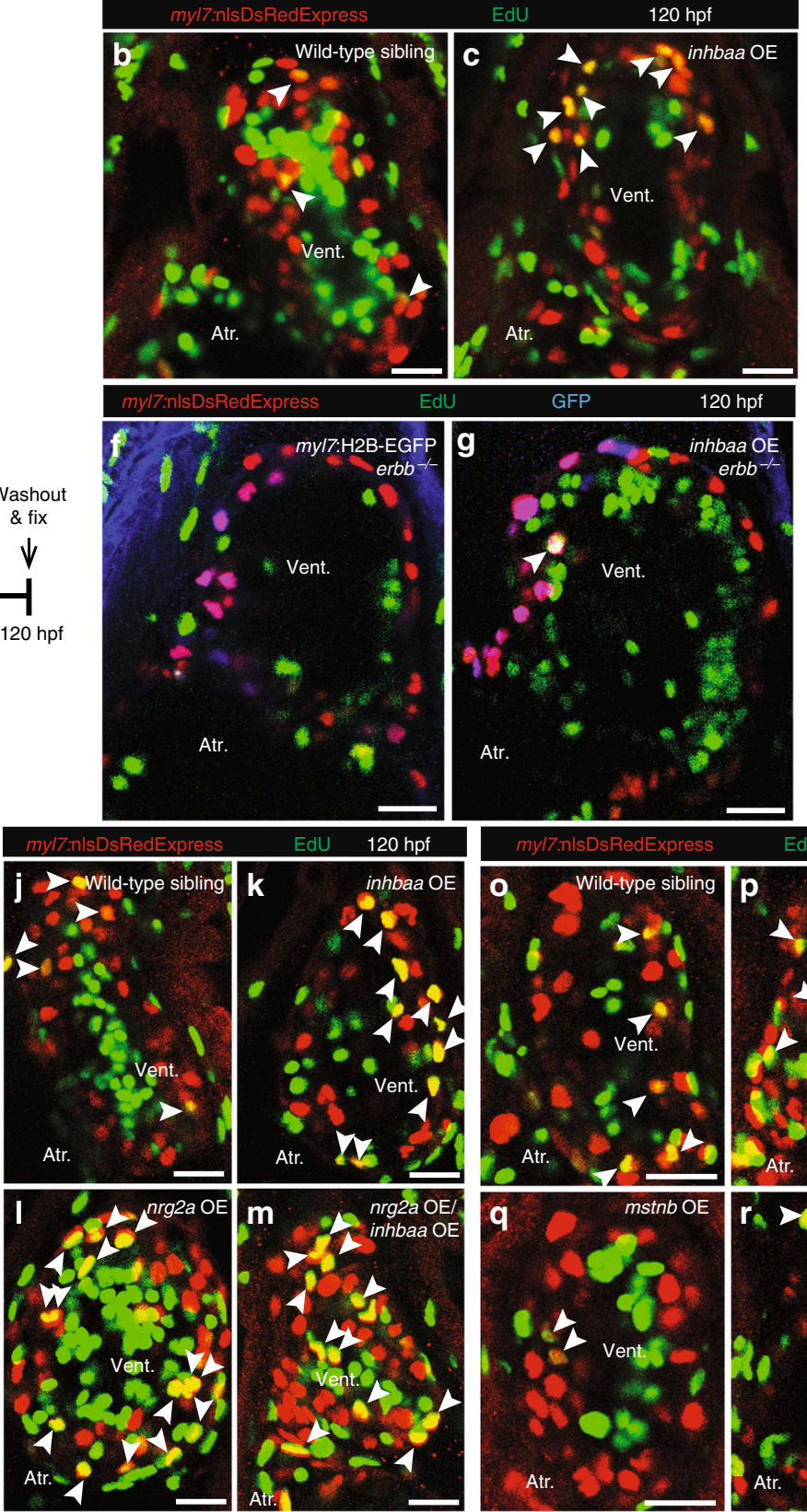
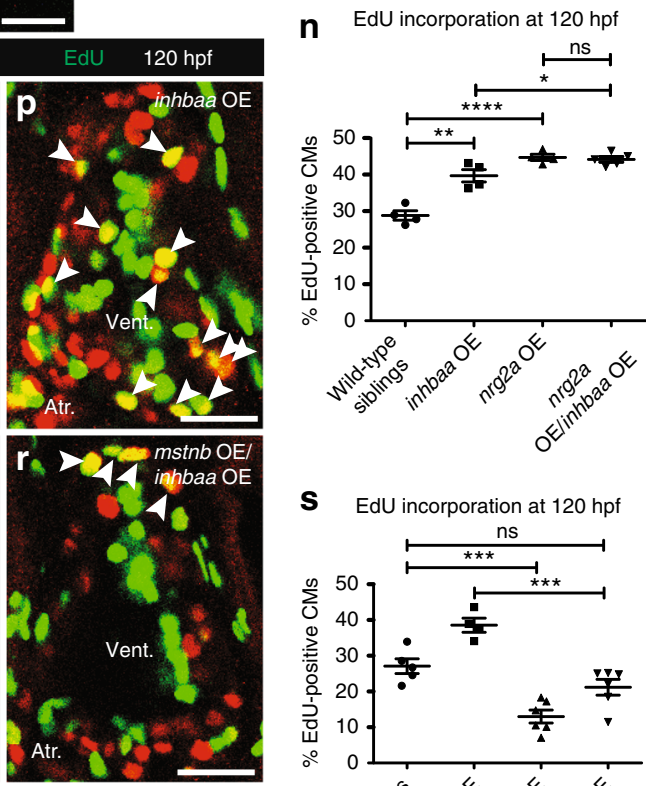

S EdU incorporation at $120 \mathrm{hpf}$

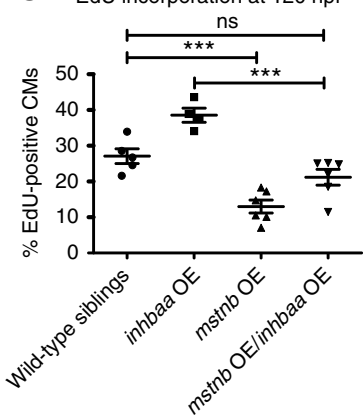

Fig. 4 inhbaa GOF promotes CM proliferation independently of ErbB2 signaling and competes with mstnb GOF. a Experimental setup of EdU treatment, followed by fixation. b, c Tg(myl7:nlsDsRedExpress) hearts of wild-type sibling and inhbaa OE larvae at 120 hpf; $\alpha$-DsRed (red), EdU (green). White arrowheads point to proliferating CMs $\left(E_{d U}{ }^{+} / \operatorname{DsRed}^{+}\right)$. d Quantification of CM proliferation in wild-type sibling $(n=6)$ and inhbaa OE $(n=6)$ ventricles at 120 hpf. e Experimental setup of injections, EdU exposure, followed by fixation. f, g Tg(myl7:nlsDsRedExpress) hearts of myl7:H2B-EGFP and myl7:inhbaa-2AH2B-EGFP(inhbaa OE) injected erbb ${ }^{-/-}$larvae at $120 \mathrm{hpf} \alpha$-DsRed (red), $\alpha$-GFP (blue), EdU (green). White arrowheads point to proliferating CMs (EdU $\left.{ }^{+} / \mathrm{DsRed}^{+} / \mathrm{GFP}^{+}\right)$. h Quantification of CM proliferation in myl7:H2B-EGFP $(n=7)$ and inhbaa OE $(n=7)$ injected ventricles at 120 hpf. i Experimental setup of EdU treatment, followed by fixation. j-m Tg(myl7:nlsDsRedExpress) hearts of wild-type sibling, inhbaa OE, nrg2a OE, and nrg2a OE/inhbaa OE larvae at 120 hpf; $\alpha$-DsRed (red), EdU (green). White arrowheads point to proliferating CMs (EdU $/$ DsRed $^{+}$). $\mathbf{n}$ Quantification of CM proliferation in wild-type sibling ( $n$ $=4)$, inhbaa OE $(n=4)$, nrg2a OE $(n=4)$, and nrg2a OE/inhbaa OE $(n=5)$ ventricles at 120 hpf. o-r Tg(myl7:n/sDsRedExpress) hearts of wild-type sibling, inhbaa OE, mstnb OE, and mstnb OE/inhbaa OE larvae at $120 \mathrm{hpf} ; \alpha$-DsRed (red), EdU (green). s Quantification of CM proliferation in wild-type sibling ( $n=$ $5)$, inhbaa OE $(n=4)$, mstnb OE $(n=6)$, and mstnb OE/inhbaa OE $(n=6)$ ventricles at $120 \mathrm{hpf}$. All cell counts were performed on non-overlapping confocal planes (thickness, $1 \mu \mathrm{m}$ ) (data are mean \pm s.e.m., ns: no significant changes observed, ${ }^{\star} P \leq 0.05,{ }^{\star \star} P \leq 0.01,{ }^{\star \star \star} P \leq 0.001$ and ${ }^{\star \star \star \star} P \leq 0.0001-$ Student's $t$ test, two-tailed). Scale bars, $20 \mu \mathrm{m}$. vent., ventricle; atr., atrium 
background. Measuring EdU incorporation (Fig. 4i) followed by genotyping, we found that mstnb $\mathrm{OE}$ reduces myocardial EdU incorporation induced by inhbaa $\mathrm{OE}$ back to wild-type levels (Fig. 4o-s). Hence, our results suggest that Mstnb and Inhbaa compete in controlling CM cell cycle progression.

mstnb and inhbaa overexpression activate distinct Smads. We further aimed to decipher the molecular mechanisms underlying the differential regulation of $\mathrm{CM}$ proliferation and therefore, cardiac regeneration by mstnb and inhbaa. Myostatin and Activin have been reported to act through the TGF- $\beta$ signaling cascade, leading to the phosphorylation of Smad2 and Smad3 ${ }^{21,22,41}$. Smad2, along with Smad4 and transcription factors such as FAST1/2, binds to the activin response elements (ARE) present in the promoter regions of target genes ${ }^{42-44}$. Similarly, Smad3 binds to the Smad-binding elements (SBE) present in the promoter a

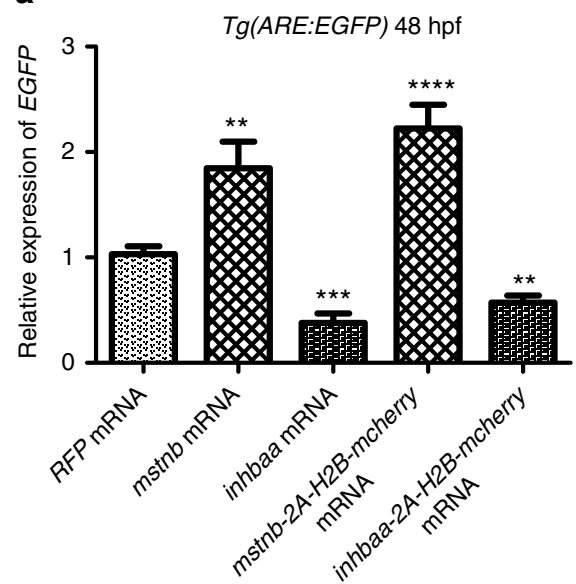

14 dpci
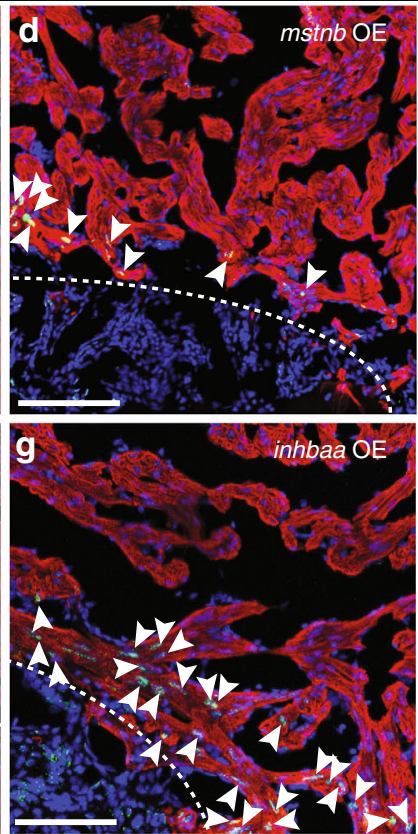

h

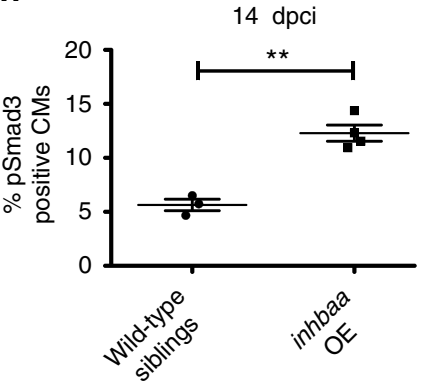

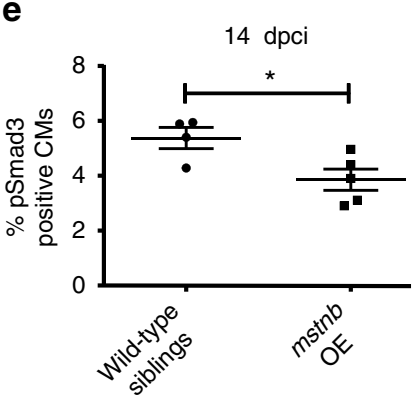
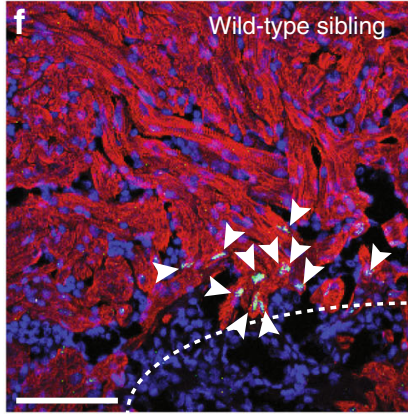

e

\section{b}
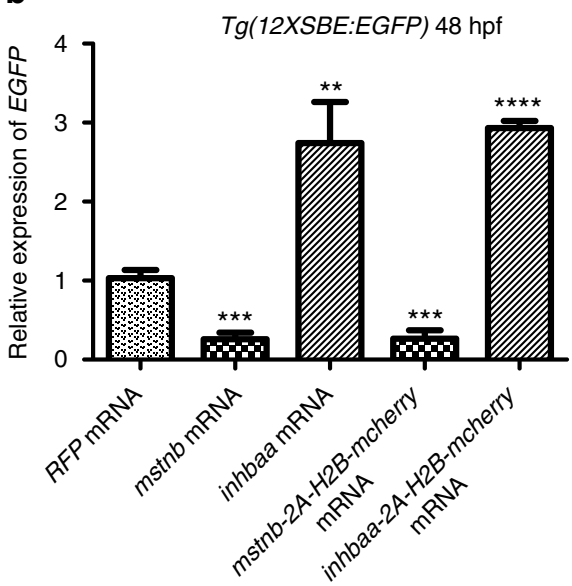

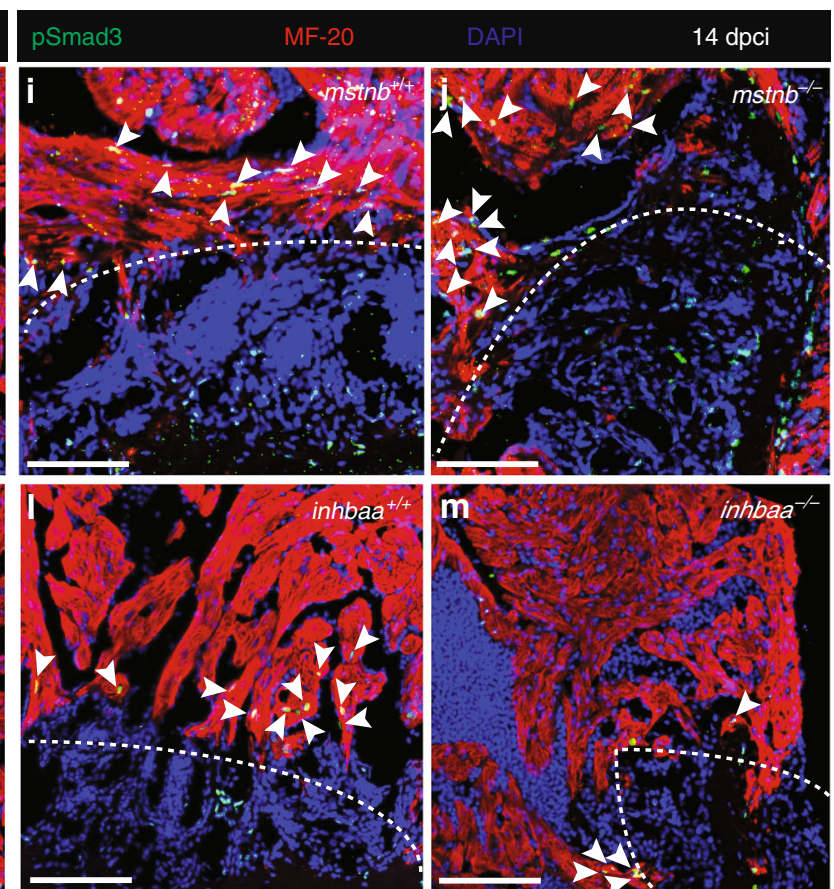

k

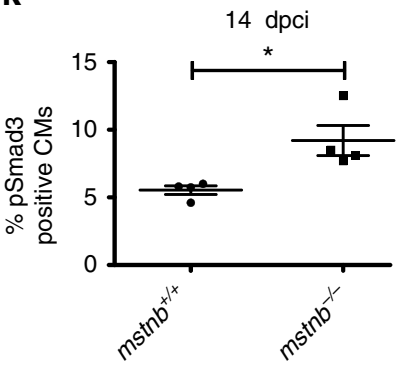


region of target genes ${ }^{45,46}$. To identify potentially distinct transcriptional target genes of Mstnb and Inhbaa signaling, we used the published Smad 2 reporter line, $\mathrm{Tg}(A R E: E G F P)^{47}$ and Smad3 reporter line, $\operatorname{Tg}(12 X S B E: E G F P)^{48}$. After injecting mstnb-2A$H 2 B$-mcherry and inhbaa-2A-H2B-mcherry mRNA in $\mathrm{Tg}(A R E$ : $E G F P)$ and $T g(12 X S B E: E G F P)$ embryos at the one-cell stage, we quantified EGFP mRNA expression at $48 \mathrm{hpf}$ by RT-qPCR. Our results show a robust induction of $S m a d 2$ reporter expression and a downregulation of Smad3 reporter expression after mstnb-2A$H 2 B$-mcherry mRNA injections (Fig. 5a, b). Conversely, we found that inhbaa-2A-H2B-mcherry mRNA injections induced Smad3 reporter activity and suppressed EGFP mRNA expression in the Smad2 reporter system (Fig. 5a, b). To make sure that our P2Alabeling strategy did not interfere with protein function and overall specificity, the aforementioned experiments were also performed with non-tagged wild-type versions leading to results identical in magnitude and specificity (Fig. 5a, b). This differential regulation of the activities of distinct Smad responsive elements by mstnb and inhbaa might account for their different influence on CM proliferation.

To further validate these results, we investigated the transcriptional response of several known TGF- $\beta$ targets in response to inhbaa or mstnb OE. Several TGF- $\beta$ target genes have been reported to be specific targets of Smad2, including Goosecoid $(G s c)^{44}$ and Mix. $2^{43}$ or Smad3, including JunB ${ }^{45}$ and Plasminogen activator inhibitor-1(PAI-1 $)^{46}$. We thus analyzed the expression of these target genes (along with their paralogs) in $48 \mathrm{hpf} m s t n b$ $2 A-H 2 B$-mcherry mRNA and inhbaa-2A-H2B-mcherry mRNA injected embryos, by RT-qPCR. Interestingly, we found an upregulation of Smad2 target gene expression by mstnb OE but a downregulation of their expression after inhbaa OE (Supplementary Fig. 6a-d). Conversely, we observed an upregulation of Smad3 target gene expression by inhbaa OE but a downregulation of their expression after mstnb OE (Supplementary Fig. 6e-1). We further tested whether mstnb and inhbaa OE was able to regulate the expression of these Smad target genes in the injured adult heart. By performing RT-qPCR at $4 \mathrm{dpci}$, we found that the expression of Smad2 target genes was induced in mstnb OE hearts, whereas either no significant effect or a transcriptional downregulation was observed in inhbaa OE hearts (Supplementary Fig. $7 \mathrm{a}-\mathrm{d}$ ). Conversely, inhbaa OE was able to induce the expression of Smad3 target genes in regenerating hearts, whereas their expression was either unchanged or downregulated after mstnb OE (Supplementary Fig. 7e-1). These results further suggest that Smad2 and Smad3 activities are inversely regulated by mstnb and inhbaa.

mstnb and inhbaa inversely regulate Smad3 phosphorylation. Smad3-dependent TGF- $\beta$ signaling has previously been linked to cardiac regeneration as Activin type 1 receptor inhibition caused a reduction in the number of $\mathrm{pSmad}^{+} \mathrm{CMs}$ and ultimately blocked cardiac regeneration ${ }^{31}$. Additionally, by using the same chemical inhibitor, it has been shown that the inhibition of TGF$\beta$ signaling negatively affects $\mathrm{CM}$ proliferation in zebrafish ${ }^{49}$. Thus, in order to test whether mstnb and inhbaa affect the phosphorylation of myocardial Smad3 during cardiac regeneration, we performed immunostainings for pSmad3 and MF-20 using a DAPI counterstain at $14 \mathrm{dpci}$, followed by quantification of $\mathrm{pSmad} 3^{+} \mathrm{CMs}$ proximal to injury site in the respective gain-offunction and loss-of-function genotypes. Interestingly, we found that $\mathrm{OE}$ of $m s t n b$ inhibits Smad3 phosphorylation and detected a $28 \%\left( \pm 10 \%\right.$ s.e.m.) decrease in the number of $\mathrm{pSmad}^{+} \mathrm{CMs}$ in $m s t n b$ OE compared to control (Fig. 5c-e). Conversely, OE of inhbaa induced myocardial Smad3 phosphorylation, with an increase of $118 \%$ ( $\pm 20 \%$ s.e.m.) in the number of $\mathrm{pSmad}^{+} \mathrm{CMs}$ compared to control (Fig. $5 \mathrm{f}-\mathrm{h}$ ). Notably, we also observed that $m s t n b^{-/-}$hearts show an induction of Smad3 phosphorylation in CMs, with an increase of $66 \%$ ( $\pm 21 \%$ s.e.m.) in the number of $\mathrm{pSmad}^{+} \mathrm{CMs}$ compared to control (Fig. 5i-k). Further, we detected a decline of $26 \%( \pm 7.5 \%$ s.e.m. $)$ in the number of pSmad $3^{+} \mathrm{CMs}$ in inhbaa ${ }^{-/}$compared to control (Fig. 5l-n), indicating that Inhbaa is important to induce Smad3 phosphorylation during cardiac regeneration. Overall, our data indicate that Mstnb and Inhbaa act antagonistically to one another in controlling Smad3 phosphorylation, an apparently crucial event during cardiac regeneration in zebrafish.

Smad2 and Smad3 inversely affect CM proliferation. Myocardial Smad3 phosphorylation is induced at the site of injury during cardiac regeneration ${ }^{31}$. Our data from regenerating mstnb $\mathrm{OE}$ and inhbaa ${ }^{-1-}$ hearts show that myocardial Smad3 phosphorylation proximal to the lesion was reduced (Fig. $5 c-e, 1-n$ ), as was CM proliferation. In order to test the hypothesis that Smad3 phosphorylation is directly linked to CM proliferation, we used a small molecule inhibitor, SIS3, to block TGF- $\beta$-mediated Smad3 phosphorylation ${ }^{50,51}$. By performing RT-qPCR, we first tested whether SIS3 acts as a specific inhibitor of Smad3 phosphorylation, and found that Smad3 target gene expression was downregulated in 72 hpf SIS3-treated hearts (Supplementary Fig. 8h-l), while Smad2 target gene expression remained unchanged (Supplementary Fig. 8e-g). We also analyzed the effect of the established Activin type 1 receptor inhibitor (SB431542) on Smad target gene expression, by performing RT-qPCR in $72 \mathrm{hpf}$ SB431542-treated hearts. We observed a downregulation in the expression of both Smad2 and Smad3 target genes (Supplementary Fig. $8 \mathrm{e}-\mathrm{l}$ ), confirming that this inhibitor blocks the complete TGF- $\beta$ pathway as reported previously ${ }^{52}$. Next, we treated $T g$ (myl7:nlsDsRedExpress) larvae with SIS3 and analyzed CM

Fig. 5 mstnb and inhbaa inversely regulate the activities of Smad2 and Smad3 response elements, as well as Smad3 phosphorylation. a RT-qPCR analysis for relative EGFP mRNA expression in 48 hpf $\mathrm{Tg}$ (ARE:EGFP) embryos injected with mstnb, inhbaa, mstnb-2A-H2B-mcherry, and inhbaa-2A-H2B-mcherry mRNA compared to RFP mRNA injected ( $n=2 \times 10$ embryos assessed as 2 biological and 2 technical replicates). b RT-qPCR analysis for relative EGFP mRNA expression in $48 \mathrm{hpf} \mathrm{Tg}(12 \mathrm{XSBE}: \mathrm{EGFP})$ embryos injected with mstnb, inhbaa, mstnb-2A-H2B-mcherry, and inhbaa-2A-H2B-mcherry mRNA compared to RFP mRNA injected ( $n=2 \times 10$ embryos assessed as two biological and two technical replicates). c, d Sections of wild-type sibling and mstnb OE cryoinjured hearts at 14 dpci; pSmad3 (green), $\alpha-M F-20$ (red), DAPI (blue). White dotted regions delineate the injured area. White arrowheads point to pSmad3 ${ }^{+}$CMs near the injured area. e Quantification of $\mathrm{pSmad}^{+} \mathrm{CMs}$ in wild-type sibling $(n=4)$ and mstnb OE $(n=5)$ cryoinjured hearts in the $100 \mu \mathrm{m}$ region adjacent to the injured area at $14 \mathrm{dpci} . \mathbf{f}, \mathbf{g}$ Sections of wild-type sibling and inhbaa OE cryoinjured hearts at 14 dpci; pSmad3 (green), $\alpha-M F-20$ (red), DAPI (blue). $\mathbf{h}$ Quantification of $\mathrm{pSmad} 3^{+} \mathrm{CMs}$ in wild-type sibling $(n=3)$ and inhbaa OE $(n=4)$ cryoinjured hearts in the $100 \mu \mathrm{m}$ region adjacent to the injured area at 14 dpci. i, j Sections of $m s t n b^{+/+}$and $m s t n b^{-/-}$cryoinjured hearts at 14 dpci; pSmad3 (green), $\alpha-M F-20$ (red), DAPI (blue). $\mathbf{k}$ Quantification of pSmad3 ${ }^{+}$CMs in $\mathrm{mstnb}^{+/+}(n=4)$ and $m s t n b^{-/-}(n=4)$ cryoinjured hearts in the $100 \mu \mathrm{m}$ region adjacent to the injured area at $14 \mathrm{dpci}$. I, $\mathbf{m}$ Sections of inhbaa ${ }^{+/+}$and inhbaa $^{-/-}$cryoinjured hearts at 14 dpci; pSmad3 (green), $\alpha-M F-20$ (red), DAPI (blue). $\mathbf{n}$ Quantification of pSmad3 ${ }^{+} \mathrm{CMs}^{\text {in inhbaa }}{ }^{+/+}(n=4)$ and inhbaa $-/-(n=5)$ cryoinjured hearts in the $100 \mu \mathrm{m}$ region adjacent to the injured area at $14 \mathrm{dpci}$. All cell counts were performed on three sections from each heart. Each data point on dot plot represents one heart (data are mean \pm s.e.m., ${ }^{\star} P \leq 0.05,{ }^{\star \star} P \leq 0.01,{ }^{\star \star \star} P \leq 0.001$ and ${ }^{\star \star \star \star} P \leq 0.0001-$ Student's $t$ test, two-tailed). Scale bars, $100 \mu \mathrm{m}$ 
a
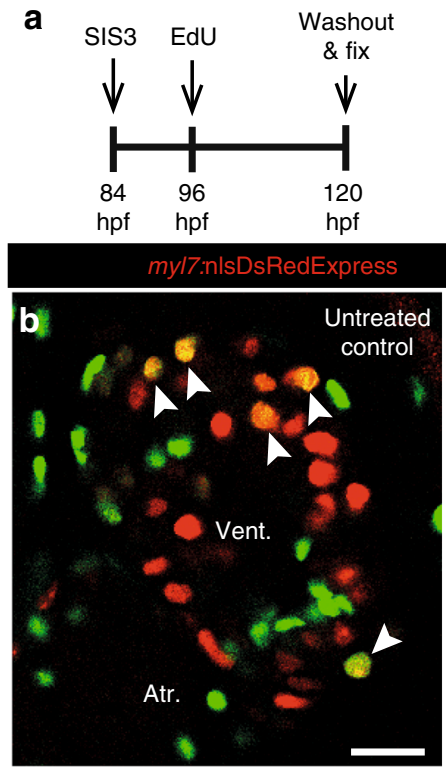

d

EdU incorporation at $120 \mathrm{hpf}$

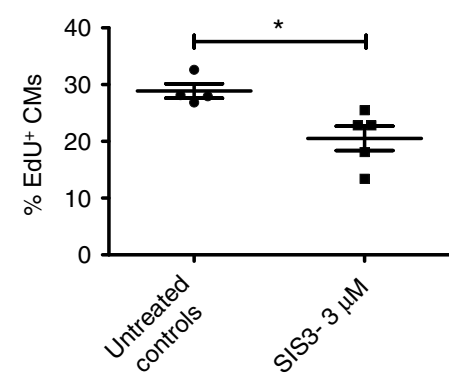

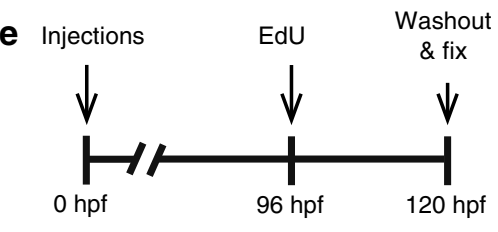
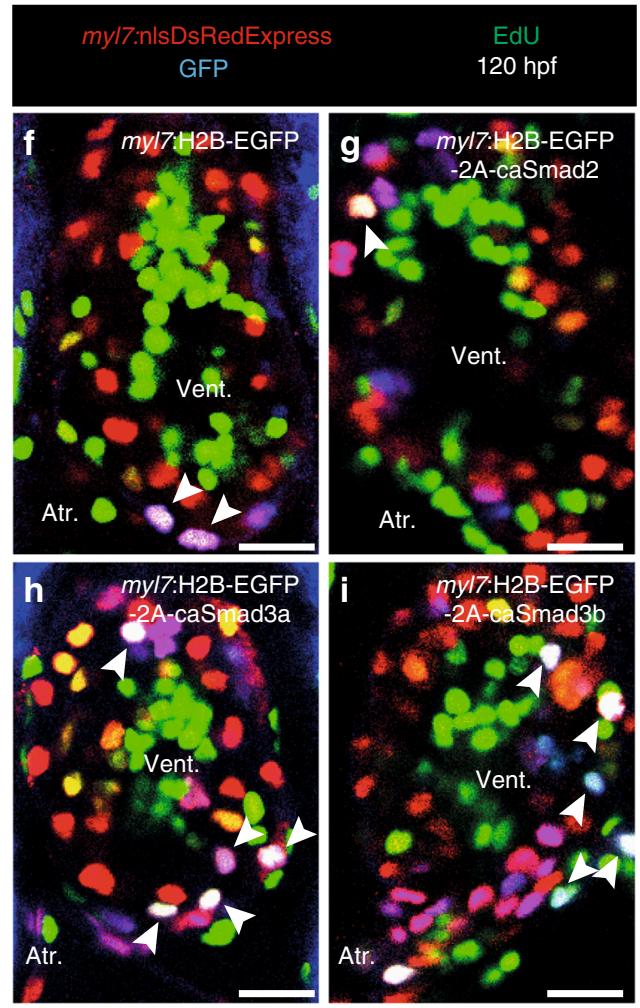

Fig. 6 CM proliferation is inversely regulated by Smad2 and Smad3. a Experimental setup of SIS3 treatment, followed by EdU treatment and fixation. b, c Tg (myl7:nlsDsRedExpress) hearts of untreated control and $3 \mu \mathrm{M}$ SIS3-treated larvae at 120 hpf; $\alpha$-DsRed (red), EdU (green). White arrowheads point to

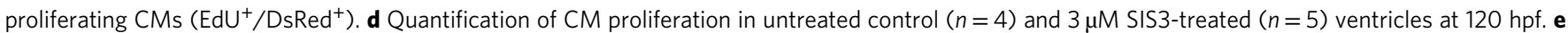
Experimental setup of injections, followed by EdU treatment and fixation. f-i $T g(m y l 7: n / s D s R e d E x p r e s s)$ hearts of myl7:H2B-EGFP, myl7:H2B-EGFP-2AcaSmad2, myl7:H2B-EGFP-2A-caSmad3a and myl7:H2B-EGFP-2A-caSmad3b injected larvae at 120 hpf; $\alpha$-DsRed (red), $\alpha$-GFP (blue), EdU (green). White arrowheads point to proliferating CMs (EdU $\left.{ }^{+} / \mathrm{DsRed}^{+} / \mathrm{GFP}^{+}\right)$. j Quantification of CM proliferation in $m y / 7: H 2 B-E G F P(n=10), \mathrm{caSmad} 2(n=10), \mathrm{caSmad} 3 a$

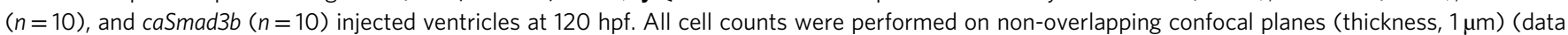
are mean \pm s.e.m., ${ }^{\star} P \leq 0.05$ and ${ }^{\star \star \star} P \leq 0.001-$ Student's $t$ test, two-tailed). Scale bars, $20 \mu m$. vent., ventricle; atr., atrium

proliferation by performing immunostaining for DsRed, followed by EdU labeling at $120 \mathrm{hpf}$ (Fig. 6a). Quantification of EdU incorporation in CMs of larval ventricles revealed that $3 \mu \mathrm{M}$ SIS3 was sufficient to substantially reduce the number of EdU ${ }^{+} \mathrm{CMs}$ (Fig. 6b-d), suggesting that CM proliferation relies on Smad3 phosphorylation.

To further test our model, we analyzed the effects of constitutively active (ca) Smad2, Smad3a and Smad3b on CM proliferation following mosaic OE. For this analysis, we generated constructs expressing $\mathrm{ca}$, phosphomimetic, Smads under the CMspecific $m y l 7$ promoter, namely $\mathrm{Tg}(\mathrm{myl} / \mathrm{H}: \mathrm{H}$ B-EGFP-2A-caSmad2) (caSmad2 hereafter), Tg(myl7:H2B-EGFP-2A-caSmad3a) (caSmad3a hereafter), $\operatorname{Tg}(m y l 7: H 2 B-E G F P-2 A-c a S m a d 3 b)$ (caSmad3b hereafter), and $\operatorname{Tg}(m y l 7: H 2 B-E G F P)$ as control (Supplementary Fig. 8a-d). We first tested whether the caSmad2, caSmad $3 a$, and $c a S m a d 3 b$ constructs were functional and specific, by performing RT-qPCR analysis for Smad2 and Smad3 target gene expression on hearts of $72 \mathrm{hpf}$ larvae obtained from outcrossing the caSmad2, $c a S m a d 3 a$, and $c a S m a d 3 b$ founders. We observed an upregulation in Smad2 target gene expression in caSmad2 hearts (Supplementary Fig. $8 \mathrm{e}-\mathrm{g}$ ), and an upregulation in Smad3 target gene expression in $c a S m a d 3 a$ and caSmad $3 b$ hearts (Supplementary Fig. 8h-1). After injection of the constructs at the one-cell stage, we analyzed CM proliferation by performing immunostaining for GFP and DsRed, followed by EdU labeling in $120 \mathrm{hpf} \operatorname{Tg}($ myl7:nlsDsRedExpress) larvae (Fig. 6e). Comparing $\mathrm{GFP}^{+} \mathrm{CMs}$ across all constructs, we found that CMs expressing caSmad2 showed a $50 \%$ ( $\pm 12 \%$ s.e.m.) reduction in EdU incorporation compared to CMs expressing GFP alone, whereas both caSmad $3 a$ and $c a S m a d 3 b$ expression resulted in a $70 \%( \pm 18 \%$ s.e.m. $)$ and $31 \%( \pm 12 \%$ s.e.m. $)$ increase in EdU incorporation, respectively (Fig. $6 \mathrm{f}-\mathrm{j}$ ). These results clearly indicate that Smad2 and Smad3 inversely regulate $\mathrm{CM}$ proliferation in zebrafish and could potentially explain how different TGF- $\beta$ family ligands can have opposite effects during regeneration.

Mstnb and Inhbaa signal through distinct Activin receptors. Mstn and Inhba have been described to signal through the same 
type 2 receptor complex ${ }^{21,22,41}$. We wanted to investigate how Mstnb and Inhbaa could function antagonistically if they work through the same signaling cascade. Thus, to better understand the ligand-receptor relationships, we performed combined gene knockdown and $\mathrm{OE}$ experiments. We co-injected morpholinos (MOs) for the activin type 2 receptor genes (acvr2aa, acvr2ab, $a c v r 2 b a$, and $a c v r 2 b b$ ) with mstnb-2A-H2B-mcherry mRNA or inhbaa-2A-H2B-mcherry mRNA in the $\operatorname{Smad} 2^{47}$ and $S m a d 3^{48}$ reporter lines. By performing RT-qPCR for EGFP mRNA expression, we found that co-injection of acvr2a MOs (acvr2aa and $a c v$ $r 2 a b$ ) along with mstnb-2A-H2B-mcherry mRNA did not influence the effect of mstnb mRNA injections, since Smad2 reporter expression was induced and Smad3 reporter expression was suppressed compared to control (Fig. 7a, b). In contrast, the coinjection of $a c v r 2 b$ MOs (acvr2ba and $a c v r 2 b b)$ along with mstnb$2 A-H 2 B$-mcherry mRNA significantly reduced the effect of mstnb OE on Smad 2 and Smad3 reporter activity (Fig. 7c, d). These results indicate that Mstnb has a preference for binding Acvr2b over Acvr $2 a$, as per previous studies ${ }^{41,53}$. Next, we tested the relationship between Inhbaa and Acvr2ba and Acvr2bb. We found that the coinjection of acvr2b MOs (acvr2ba and acvr2bb) with inhbaa-2A$\mathrm{H} 2 \mathrm{~B}$-mcherry mRNA did not influence the effect of inhbaa OE, as Smad2 reporter expression was suppressed and Smad3 reporter expression was induced compared to control (Fig. 7e, f). In contrast, the co-injection of acvr2a MOs (acvr2aa and acvr2ab) along with inhbaa-2A-H2B-mcherry mRNA significantly reduced the effect of a

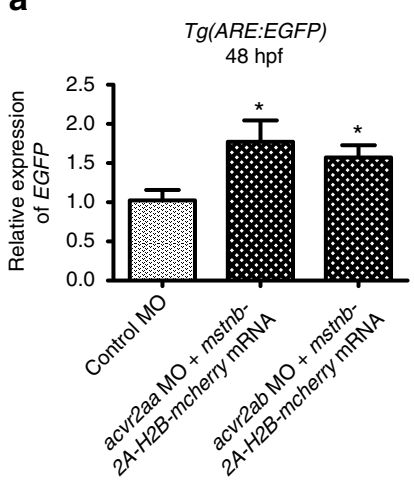

e
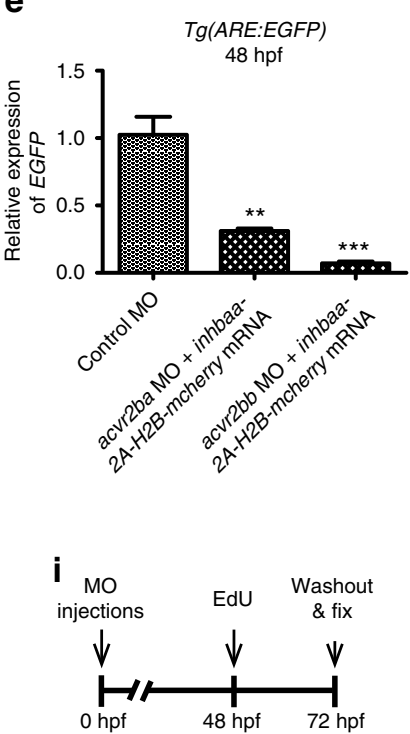

b

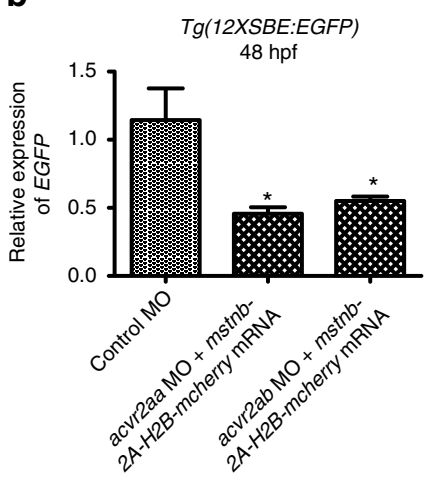

f
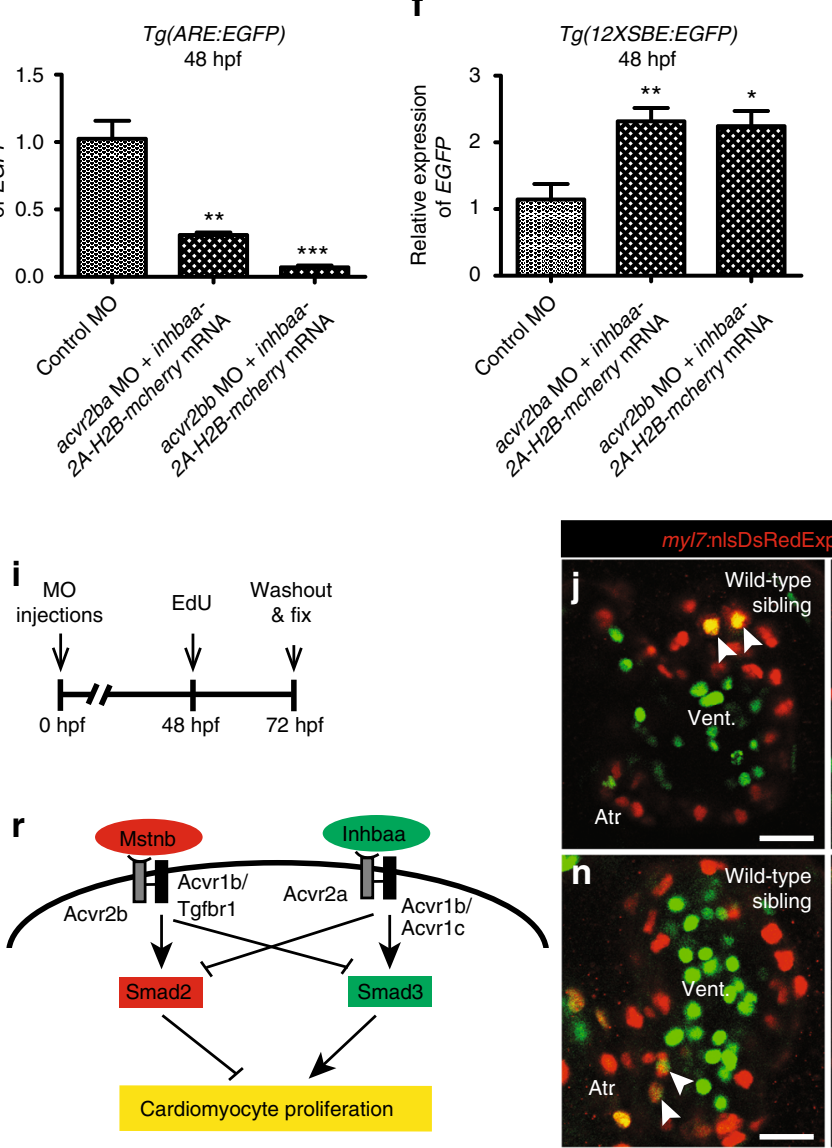

C

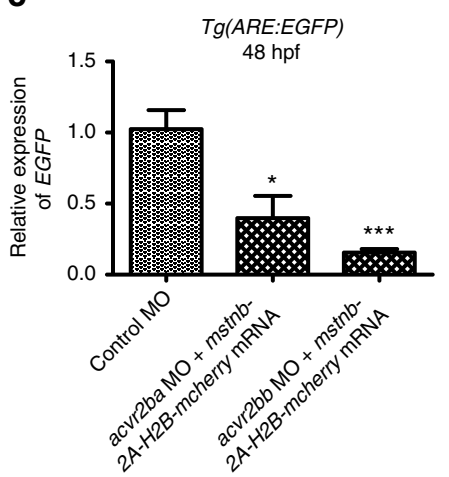

g
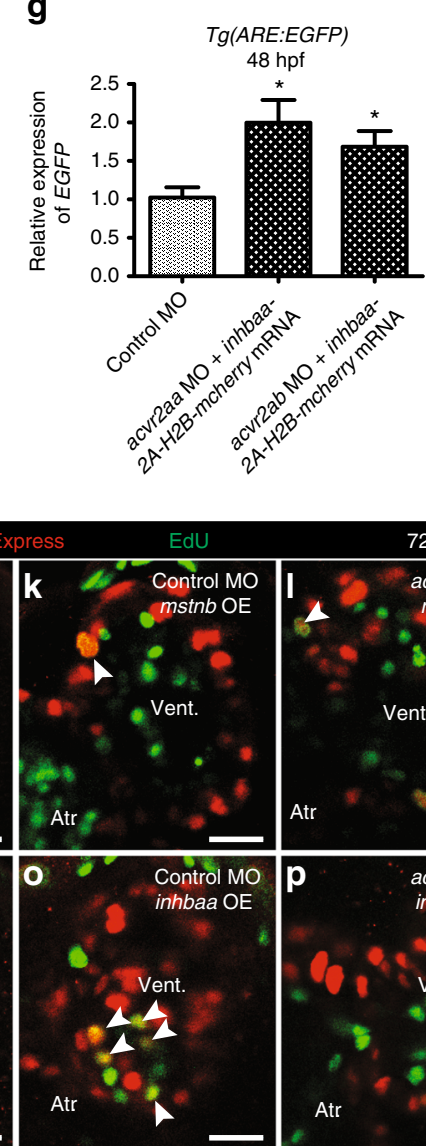

d

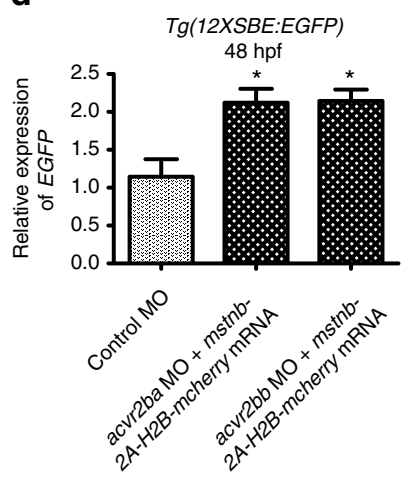

h

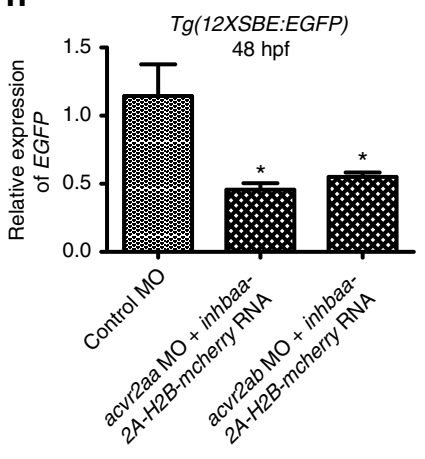

m EdU incorporation at $72 \mathrm{hpf}$
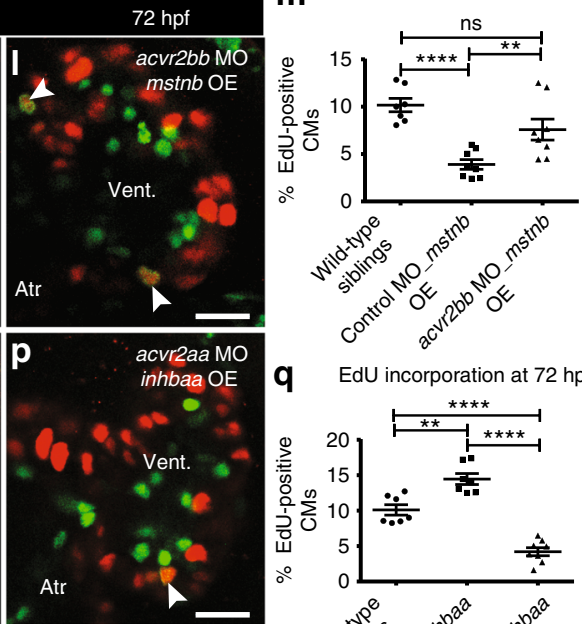

q EdU incorporation at $72 \mathrm{hpf}$

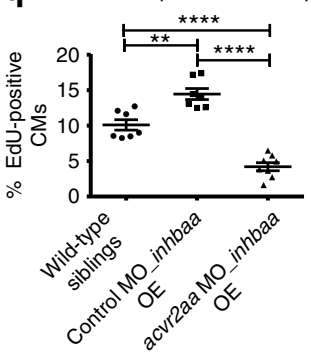


inhbaa OE on Smad2 and Smad3 reporter activity (Fig. $7 \mathrm{~g}, \mathrm{~h}$ ). These data suggest a previously unreported preference for Inhbaa to signal through Acvr2a over Acvr2b.

Finally, we also analyzed the effect of these receptor knockdowns in the CM EdU incorporation assay using our mstnb OE and inhbaa OE lines (Fig. 7i). Since its effect on Smad2 reporter expression was more significant compared to acvr2ba MO, we injected the acvr2bb MO in the mstnb OE line and assessed CM EdU incorporation at $72 \mathrm{hpf}$. We observed a significant increase of $94 \%$ ( $\pm 33 \%$ s.e.m.) in CM EdU incorporation in acvr $2 b b \mathrm{MO}-$ injected mstnb OE larvae compared to control (Fig. 7j-m). This result is in line with our RT-qPCR data that Acvr2b is a specific receptor for Mstnb, and knocking down $a c v r 2 b b$ prevents the suppression of CM proliferation by mstnb OE. Next, we injected acvr2aa MO in the inhbaa OE line and examined CM EdU incorporation at $72 \mathrm{hpf}$. Here, we observed a significant reduction of $70 \%$ ( $\pm 8 \%$ s.e.m.) in CM EdU incorporation in acvr2aa MOinjected inhbaa OE larvae compared to control (Fig. $7 \mathrm{n}-\mathrm{q}$ ). Again, this result is in line with our RT-qPCR data that Acvr2a is a specific receptor for Inhbaa, and knocking down acvr2aa prevents the induction of CM proliferation by inhbaa OE. Overall, our results suggest that Mstnb binds to Acvr2b, leading to the activation of Acvr1b/Tgfbr1, which promotes Smad2 and suppresses Smad3 activation. Inversely, Inhbaa binds to Acvr2a, leading to the activation of Acvr1b/Acvr1c, which promotes Smad3 and suppresses Smad2 activation (Fig. 7r).

\section{Discussion}

The role of TGF- $\beta$ signaling in organ regeneration and pathology remains unclear as seemingly contradictory results have been reported. TGF- $\beta$ signaling is required for the formation of the wound epithelium and cell proliferation during Xenopus tail regeneration $^{54}$, while its loss has been associated with an expansion of progenitor cells in the regenerating mammalian liver $^{55}$. Furthermore, TGF- $\beta$ signaling has been implicated in the pathogenesis of cardiac remodeling and fibrosis after pressure overload in mammals ${ }^{30}$, while the treatment of adult zebrafish with a TGF- $\beta$ receptor inhibitor blocked cardiac regeneration ${ }^{31}$. Thus, because of its highly diverse and seemingly contradictory functions across various cell types and organisms, we wanted to dissect the role of different TGF- $\beta$ family members in cardiac regeneration by performing ligand-specific genetic manipulations.

Here, we have identified the opposing expression response of two TGF- $\beta$ family ligand encoding genes, mstnb and inhbaa. From subsequent genetic studies we have revealed their contrasting roles in regulating cardiac regeneration and CM proliferation in zebrafish. Our study shows that expression of mstnb, a negative regulator of skeletal muscle growth ${ }^{32,33}$, is rapidly and continuously downregulated during cardiac regeneration in the adult zebrafish heart. However, this observation contrasts previous findings in mammals which show a rapid and significant upregulation of MSTN post $\mathrm{MI}^{25}$. We therefore hypothesized that $m s t n b$ downregulation in zebrafish following cardiac injury facilitates cardiac regeneration, whereas elevated MSTN levels in the injured mammalian heart could possibly inhibit the process of regeneration. Supporting our hypothesis, we observed that loss of $m s t n b$ positively affects physiological CM proliferation and cardiac regeneration. Contrarily, mstnb $\mathrm{OE}$ in $\mathrm{CMs}$ led to a significant decline in $\mathrm{CM}$ proliferation, reduced regeneration and compromised scar clearance after injury. Overall, our data strongly support that mstnb downregulation is important during cardiac regeneration, facilitating CM proliferation.

In contrast, we have found that inhbaa expression was upregulated in response to cardiac injury during the early stages of myocardial regeneration. Further, the loss of inhbaa strongly correlated with reduced scar clearance and CM proliferation within the proximity of the injury site. Intriguingly, CM-specific inhbaa OE not only enhanced cardiac regeneration, but also resulted in cardiomegaly as a consequence of increased CM proliferation and hypertrabeculation, even in the absence of cardiac injury. The cardiac hypertrabeculation phenotype of the inhbaa OE fish is reminiscent of that observed in mice mutant for FKBP $12^{56}$, a negative regulator of the TGF- $\beta$ family ${ }^{57}$, suggesting a conserved function of TGF- $\beta$ signaling in cardiac development and possibly CM proliferation. Recent reports have identified Nrg as a potent mitogen in fish and mammals ${ }^{4,14,17}$. We performed epistasis experiments to test whether inhbaa-induced CM proliferation depends upon Nrg-ErbB signaling. These experiments revealed that Inhbaa stimulates $\mathrm{CM}$ proliferation independently of ErbB receptor activity, indicating that similar to Nrg, Inhbaa has the potential to induce CM proliferation directly. However, simultaneous OE of $n r g 2 a$ and inhbaa did not yield a higher CM proliferative index than OE of $n r g 2 a$ alone. Possibly, stimulation of CM proliferation becomes saturated by $n r g 2 a$ OE alone, as suggested by the minimal variability observed across the different specimens. Alternatively, Nrg2a signaling might interfere with the potential of Inhbaa to promote CM proliferation. Indeed, stimulation of the MAPK signaling cascade by oncogenic mutations in Ras or by EGF receptor stimulation mediates the phosphorylation of specific residues in the linker region of $\operatorname{Smad} 2 / 3^{58}$. Phosphorylation of this linker region was shown to inhibit TGF$\beta$-induced C-terminal phosphorylation and nuclear accumulation of Smads, attenuating the transcriptional activation of their target genes $^{58}$. Interestingly, inhbaa is upregulated after cardiac injury, both in mammals ${ }^{26}$ and zebrafish, but potentially the timing and

\footnotetext{
Fig. 7 Mstnb and Inhbaa work through distinct Activin type 2 receptors to regulate CM proliferation. a-d RT-qPCR analysis for relative EGFP mRNA expression in $48 \mathrm{hpf} \mathrm{Tg}$ (ARE:EGFP) and Tg(12XSBE:EGFP) embryos injected with acvr2aa MO/mstnb-2A-H2B-mcherry mRNA, acvr2ab MO/mstnb-2A-H2Bmcherry mRNA, acvr2ba MO/mstnb-2A-H2B-mcherry mRNA, and acvr2bb MO/mstnb-2A-H2B-mcherry mRNA compared to control MO injected ( $n=2 \times 10$ embryos assessed as two biological and two technical replicates). e-h RT-qPCR analysis for relative EGFP mRNA expression in 48 hpf Tg(ARE:EGFP) and Tg (12XSBE:EGFP) embryos injected with acvr2ba MO/inhbaa-2A-H2B-mcherry mRNA, acvr2bb MO/inhbaa-2A-H2B-mcherry mRNA, acvr2aa MO/inhbaa-2A$\mathrm{H} 2 \mathrm{~B}$-mcherry mRNA, and acvr2ab MO/inhbaa-2A-H2B-mcherry mRNA compared to control MO-injected $(n=2 \times 10$ embryos assessed as two biological and two technical replicates). i Experimental setup of injections, followed by EdU treatment and fixation. $\mathbf{j}-\mathbf{I} \mathrm{Tg}$ (myl7:n/sDsRedExpress) hearts of wild-type sibling, control MO-injected mstnb OE and acvr2bb MO-injected mstnb OE larvae at 72 hpf; $\alpha$-DsRed (red), EdU (green). White arrowheads point to proliferating $\mathrm{CMs}\left(\mathrm{EdU}^{+} / \mathrm{DsRed}^{+}\right) \cdot \mathbf{m}$ Quantification of CM proliferation in wild-type sibling $(n=7)$, control MO-injected mstnb OE $(n=8)$ and $a c v r 2 b b \mathrm{MO}$-injected mstnb OE $(n=8)$ ventricles at 72 hpf. $\mathbf{n}$-p $T g$ (myl7:nlsDsRedExpress) hearts of wild-type sibling, control MO-injected inhbaa OE, and acvr2aa MO-injected inhbaa OE larvae at $72 \mathrm{hpf} ; \alpha$-DsRed (red), EdU (green). q Quantification of CM proliferation in wild-type sibling $(n=7)$, control MO-injected inhbaa OE ( $n$ $=7$ ) and acvr2aa MO-injected inhbaa OE $(n=8)$ ventricles at $72 \mathrm{hpf}$. $\mathbf{r}$ Model of ligand-receptor relationship: Mstnb binds to Acvr2b, leading to the activation of Acvr1b/Tgfbr1, which promotes Smad2 and suppresses Smad3 activation. Inversely, Inhbaa binds to Acvr2a, recruiting Acvr1b/Acvr1c, thereby inducing Smad3 and suppressing Smad2 activation. This process is followed by the differential regulation of CM proliferation by Smad2 and Smad3. All cell counts were performed on non-overlapping confocal planes (thickness, $1 \mu \mathrm{m}$ ) (data are mean \pm s.e.m., ns: no significant changes observed, ${ }^{\star} P \leq 0.05$, ${ }^{\star \star} P \leq 0.01,{ }^{\star \star \star} P \leq 0.001$ and ${ }^{\star \star \star \star} P \leq 0.0001-$ Student's $t$ test, two-tailed). Scale bars, $20 \mu \mathrm{m}$. vent., ventricle; atr., atrium
} 
levels of its induction do not allow it to induce regeneration in the mammalian heart. In mammals, prolonged upregulation of $I N H B A$ has been associated with induction of fibrosis post MI, leading to cardiac remodeling and failure ${ }^{26}$. We speculate that a transient upregulation of INHBA in injured hearts, as observed in zebrafish, along with a rapid inactivation of MSTN could be beneficial to stimulate CM proliferation while prolonged INHBA expression has negative consequences. Indeed, several of our inhbaa OE animals showed signs of cardiomyopathy and heart failure past 6 months of age, an effect that could be potentiated by the presence of a higher number of myofibroblasts and other nonmyocardial cells in the mammalian heart ${ }^{59}$.

Canonically, TGF- $\beta$ family ligands including Myostatin and Activin signal through Activin type 2 and type 1 receptors leading to the C-terminal phosphorylation of Smad2 and Smad3. Mechanistically, it has been reported that Myostatin has a binding preference for the Activin type 2 receptor Acvr2b compared to Acvr2a ${ }^{41,53}$. We experimentally validated that not only Mstnb, but also Inhbaa, have different binding affinities for Activin type 2 receptors and inversely modulate the activities of Smad2 and Smad3. Smad2 and Smad3 have highly homologous MH1 and MH2 domains; however, the MH1 domain of Smad2 has 30 extra amino acids preventing its direct binding to DNA, unlike Smad3 which can directly bind to target DNA sequences ${ }^{60}$. These structural differences in Smad2 and Smad3 may account for differences in their functions. Several recent studies have revealed the antagonistic effects of Smad2 and Smad3 during multiple cellular processes such as blastema formation in regeneration, tumor angiogenesis, and neurogenesis ${ }^{51,61,62}$. Similarly, our data suggest that constitutively active Smad2 and Smad3 act antagonistically to one another in regulating CM proliferation. Additionally, we identified differential effects of mstnb and inhbaa OE on myocardial Smad3 phosphorylation in the regenerating heart, suggesting that indeed these two ligands are inversely affecting Smad3 phosphorylation which seemingly mediates CM proliferation during cardiac regeneration. Taken together, we identified opposite functions for the two TGF- $\beta$ family ligands, Mstnb and Inhbaa, during cardiac regeneration and similarly, for their downstream effectors Smad2 and Smad3. In addition, the Nrg-ErbB independent mitogenic activity of Inhbaa may provide new avenues towards developing alternative strategies in the treatment of patients post MI.

\section{Methods}

Zebrafish. Procedures involving animals were approved by the veterinary department of the Regional Board of Darmstadt.

Zebrafish husbandry. All zebrafish husbandry was performed under standard conditions in accordance with institutional (MPG) and national ethical and animal welfare guidelines. The characterized mutant and transgenic lines erbb2 ${ }^{\text {st6163 }}, \mathrm{Tg}$ (-0.8myl7:nlsDsRedExpress)hsc4 ${ }^{64}, \mathrm{Tg}$ (myl7:nrg2a202-p2a-tdTomato)bns140 ${ }^{17}, \mathrm{Tg}$ (ARE:EGFP)fci100 ${ }^{47}$, and $\operatorname{Tg}(12 X S B E: E G F P) i a 16^{48}$ were used in this study.

Cryoinjury. To perform cryoinjury ${ }^{7}$, adult zebrafish (3-6 mpf) were anaesthetized in $0.016 \%$ tricaine and placed on a wet sponge with their ventral side up. An incision was made through the chest to access the heart and a precooled cryoprobe was applied to the ventricular apex till the cryoprobe thawed. Later, the fish were recovered by transferring them to the fresh water.

Microarray expression profiling. Total RNA was isolated from $\sim 6 \mathrm{mpf}$ sham operated and cryoinjured hearts 4 dpci using Trizol (Life Technologies). Dual color cDNA labeling and hybridization was performed by MOgene (commercial service) using the Agilent Zebrafish (V3) $4 \times 44 \mathrm{~K}$ platform. Microarray raw and normalized data have been submitted to NCBI-GEO under the accession number GSE89259.

LMD. LMD (LMD-6000, Leica) was performed on adult zebrafish heart cryosections to dissect the wall and trabecular tissues separately for RNA extraction.
Larval heart extraction. To perform heart extraction ${ }^{65}, 72 \mathrm{hpf}$ larvae expressing GFP under CM-specific myosin, light chain 7, regulatory ( $m y l 7)$ promoter were anesthetized and transferred to $1.5 \mathrm{ml}$ microfuge tube, followed by washing three times with embryo disruption medium (EDM) and resuspension in EDM. Further, $1 \mathrm{ml}$ of EDM containing larvae was drawn into the 19-gauge needle and ejected back into the microfuge tube 30 times at the rate of $1 \mathrm{~s}$ per syringe medium. Fragmented larvae were passed through $100 \mu \mathrm{m}$ nylon mesh and the flow-through was collected in the petri dish. Later, the flow-through was passed through $40 \mu \mathrm{m}$ nylon mesh. Next, the mesh was inverted and the retained material was washed off with $\mathrm{EDM}$ into the petri dish. Intact $\mathrm{GFP}^{+}$hearts were identified under fluorescent light and collected in fresh EDM. These hearts were further pooled in a $1.5 \mathrm{ml}$ microfuge tube, pelleted and the preparations were stored at $-80^{\circ} \mathrm{C}$ after removing the media. A pool of 30 extracted hearts was used for RNA extraction.

RT-qPCR. RNA from adult heart ventricles and 48 hpf embryos was extracted using Trizol. RNA from extracted larval hearts and LMD samples was extracted using miRNeasy Micro Kit (Qiagen), and the cDNA prepared (Maxima First Strand cDNA Synthesis Kit for RT-qPCR, Thermo Fisher Scientific) was used to perform RT-qPCR (CFX Connect Real-Time System, Biorad). The primers used for RT-qPCR are listed in Supplementary Table 2. rpl13 was used as an internal control. The Ct values of the genes in the control samples are listed in Supplementary Table 3

Generation of mutants and transgenic zebrafish. inhbaa and mstnb mutants were generated using TALEN-induced mutagenesis. The TALENs were designed and cloned according to Golden Gate Assembly ${ }^{66}$. The TALEN arms targeting inhbaa had the following RVDs: NN NG NN NN NG NN NN NI NN NN HD NI NN NG NN and NN HD NI NN NN NG NN HD NI NN HD NI NG NN NG. The TALEN arms targeting mstnb had the following RVDs: NN NN NI NN NI NG NI NG NI NI HD NN NN HD NN HD and HD NN HD NG NG NG HD HD NG HD HD NN NG NN NN HD. The TALEN mRNA were injected in one-cell stage embryos and mutant alleles, $m s t n b^{b n s 5}$ and $i n h b a a^{b n s 37}$ were recovered by performing High Resolution Melt Analysis, using the following primers: inhbaa F-5'-AGAGCGAGGACGAGGGAG-3', inhbaa R-5'-GTGTG TGATGTTGGGTCGCT- $3^{\prime}$ and $m s t n b$ F- $5^{\prime}$-GTGTATTAATTGCATGTGG TCCAG-3', mstnb_R-5'-GAACACTGCTCGCTTTCCTC-3'. The F1 heterozygous animals were intercrossed to raise $\mathrm{F} 2$ adults, which were used in the experiments.

For generating OE transgenes under the control of $m y l 7$ promoter, the CDS of $m s t n b$ and inhbaa was PCR amplified and fused with a self-cleaving peptide $2 \mathrm{~A}$ and H2B-EGFP, using Cold Fusion technology (System Biosciences, CA, USA). A total of $18 \mathrm{pg}$ of each of these constructs were co-injected with $20 \mathrm{pg}$ Tol 2 mRNA into one-cell stage embryos. The transgenic fish obtained were named as $\mathrm{Tg}(\mathrm{myl}$ : mstnb-2A-H2B-EGFP)bns145 and Tg(myl7:inhbaa-2A-H2B-EGFP)bns146. The founders were outcrossed with $T g(-0.8 m y l 7: n l s D s R e d E x p r e s s) h s c 4$ to generate stable lines, which were used in the experiments.

mRNA overexpression. Full length mstnb and inhbaa CDS was amplified from cDNA and cloned into pcDNA3.1. myl7:mstnb-2A-H2B-mcherry and myl7:inhbaa$2 A-H 2 B$-mcherry constructs were generated using Cold Fusion technology. For mRNA synthesis, mstnb-2A-H2B-mcherry and inhbaa-2A-H2B-mcherry CDS was cloned into pcDNA3.1. mRNA was synthesized using the mMESSAGE mMACHINE kit and $100 \mathrm{pg}$ of each mRNA was injected into $T g(A R E: E G F P)$ and $T g$ (12XSBE:EGFP) embryos at one-cell stage.

Generation of constitutively active constructs. Constitutively active versions of Smad2, Smad3a, and Smad3b were generated by site-directed mutagenesis of their C-terminal serines to aspartic acids in the SSXS phosphorylation motifs (phosphomimetic mutation $)^{67}$. By using Cold Fusion technology, these constitutively active versions were cloned into a Tol2 vector under the control of $m y l 7$ promoter, $\mathrm{Tg}$ (myl7:H2B-EGFP-2A-caSmad2), Tg(myl7:H2B-EGFP-2A-caSmad3a), Tg(myl7: $H 2 B-E G F P-2 A-c a S m a d 3 b)$. Similarly, $T g(m y l 7: H 2 B-E G F P)$ was generated as control. $20 \mathrm{pg}$ of each of these constructs were co-injected with $20 \mathrm{pg}$ Tol 2 mRNA into one-cell stage embryos.

Morpholinos. The following MOs were purchased from GeneTools (Philomath, OR) and injected at the one-cell stage at the indicated amounts in all experiments described: acvr2aa ATG MO- $1.5 \mathrm{ng}$ (5'-CCAGCTTTGTTGCAGGTCCCATTTT$\left.3^{\prime}\right)$, acvr $2 a b$ splice MO-2 ng (5'-TGGCTGCACACAAACACAGATTAAT-3'), $a c v r 2 b a$ ATG MO-1 ng (5'-TGAGCAGAGAAGCGAACATATTCCT-3'), $a c v r 2 b b$ ATG MO- $0.5 \mathrm{ng}\left(5^{\prime}\right.$-AGCCAGCCAGGGAACAAACATATTC-3') and control MO-concentrations similar to experimental MOs $\left(5^{\prime}\right.$-CCTCTTACCTCAGTTACAATTTATA-3'). All doses were determined as optimal by titration (no toxic effects were observed).

Histology and in situ hybridization. The hearts were fixed in $4 \%$ paraformalde hyde (PFA) and $7 \mu \mathrm{m}$ thick paraffin sections or $12 \mu \mathrm{m}$ thick cryosections were obtained. For H\&E staining, the cryosections were stained with acidic hemalum (Waldeck) for $10 \mathrm{~min}$, washed in running tap water for $2 \mathrm{~min}$ and rinsed in deionized water. Further, the sections were stained with eosin (Waldeck) for $6 \mathrm{~min}$, dehydrated in $100 \%$ ethanol, cleared in xylene and mounted in entellan (Merck) 
For AFOG staining, paraffin sections were fixed with Bouin's solution overnight at room temperature (RT) and stained according to the manufacturer's instructions (Gennova), without hematoxylin solution. To perform in situ hybridization ${ }^{68}$, cryosections were permeabilized in $5 \mu \mathrm{g} \mathrm{ml}^{-1}$ proteinase $\mathrm{K}$ (Roche) for $15 \mathrm{~min}$ at RT, followed by acetylation for $2 \mathrm{~min}$ and pre-incubation in hybridization buffer for $3 \mathrm{~h}$ at $70^{\circ} \mathrm{C}$. Later, the sections were incubated with DIG-labeled RNA antisense probes overnight at $70^{\circ} \mathrm{C}$. Next, the sections were washed and incubated with alkaline phosphatase-conjugated anti-digoxigenin antibody (Roche) overnight at 4 ${ }^{\circ} \mathrm{C}$. Finally, after washing, the signal was detected with NBT-BCIP staining solution (Roche). Probes for in situ hybridization were generated by using the following primer sequences: $m s t n b \_$insitu_F-5'-CCCATTGTTCAAGTAGATCGG-3', mstnb_insitu_R-5'-ATTGTCCATTCCCGAGTCCA-3', inhbaa_insitu_F-5'-ATC ATCACGT TCGCTGAAACC-3' and inhbaa_insitu_R-5'-GAGAGTTCGTCTTG AGGCAG-3'.

Immunofluorescence. To perform immunofluorescence ${ }^{7}$, cryosections were fixed in $4 \%$ PFA for $15 \mathrm{~min}$, followed by antigen retrival for $20 \mathrm{~min}$ at $95^{\circ} \mathrm{C}$ (for PCNA antibody staining), permeabilization in $0.5 \%$ Triton-X for $15 \mathrm{~min}$ at RT and incubation in blocking buffer (1\% BSA in PBS) for $1 \mathrm{~h}$ at RT. Later, the sections were incubated in primary antibody overnight at $4^{\circ} \mathrm{C}$. Next, after washing, the sections were incubated with secondary antibody overnight at $4{ }^{\circ} \mathrm{C}$. Finally, the immunostained slides were mounted with mowiol for imaging. To perform immunofluorescence ${ }^{69}$, whole-mount larvae were fixed in $4 \%$ PFA overnight at $4^{\circ}$ $\mathrm{C}$, followed by incubation in permeabilization solution $(0.3 \%$ Triton-X, $1 \%$ DMSO, $1 \%$ BSA, and $0.1 \%$ Tween-20) for $3 \mathrm{~h}$ at RT. Further, the larvae were incubated in blocking solution (1\% DMSO, $2 \% \mathrm{FBS}, 1 \% \mathrm{BSA}$, and $0.1 \%$ Tween-20) for $1 \mathrm{~h}$ at RT. Next, the larvae were incubated in primary antibody overnight at $4{ }^{\circ} \mathrm{C}$. Later, after washing, the larvae were incubated in secondary antibody overnight at $4{ }^{\circ} \mathrm{C}$. Finally, the stained larvae were washed, mounted in $1.5 \%$ low melting agarose for imaging. Primary antibodies used for immunofluorescence were anti-PCNA at 1:200 (mouse; Dako), anti-DsRed at 1:300 (rabbit, Clontech), anti-pSmad3 at 1:200 (rabbit; Abcam), anti-MF-20 at 1:500 (mouse; eBioscience), anti-GFP at 1:500 (chicken, Aves Labs) and anti-N2.261 at 1:50 (mouse, H.M. Blau, Developmental Studies Hybridoma Bank). Secondary antibodies were used at 1:500 (Life Technologies)

Imaging and quantification. The immunostained slides and larvae were imaged at $\times 20$ magnification and $\times 40$ magnification, respectively using LSM700/LSM800 confocal microscopes (Zeiss). After imaging, the acquired confocal z-stacks were processed and cell counting was performed with ZEN (Zeiss), Fiji, and Imaris (Bitplane) softwares. Bright field images were obtained with stereomicroscopes (SMZ25, Nikon and Stereodiscovery V8, Zeiss). Ventricular, atrial and eye sizes were measured by using ZEN software (apex to base). Ventricular wall thickness was also measured by using ZEN software, by taking the average of three regions near the apex.

Genotyping. For genotyping the immunostained larvae obtained by crossing different transgenic backgrounds, PCR was performed on genomic DNA using the primers listed in Supplementary Table 4. erbb2 ${ }^{s t 61}$ mutants were genotyped ${ }^{63}$ by using PCR-based restriction fragment length polymorphism analysis. PCR products obtained from genomic DNA samples (using primer pairs listed in Supplementary Table 4) were cut with BsrGI, resulting in a genotype-specific DNA band pattern.

EdU treatment. For EdU incorporation analysis, adult fish were anaesthetized with $0.016 \%$ tricaine and $200 \mu \mathrm{g}$ of EdU (Invitrogen) was injected intraperitoneally. The hearts were sampled after 3 days of EdU incubation and fixed in 4\% PFA. $1 \mathrm{mM}$ EdU was used to incubate embryos from $48 \mathrm{hpf}$ to $72 \mathrm{hpf}$ and larvae from $96 \mathrm{hpf}$ to $120 \mathrm{hpf}$, followed by fixation in 4\% PFA. EdU labeling was performed according to the manufacturer's protocol (Invitrogen).

ErBb2 and TGF- $\beta$ signaling inhibitor treatments. The ErBb2 signaling inhibitor (PD168393, Calbiochem $)^{70}$, Smad3 phosphorylation inhibitor (SIS3, Calbiochem) 50 and Activin type 1 receptor inhibitor (SB431542, Calbiochem) ${ }^{52}$ were used to treat the embryos or larvae. The embryos were treated with $3 \mu \mathrm{M}$ SIS3 and $10 \mu \mathrm{M}$ SB431542 from $36 \mathrm{hpf}$ to $72 \mathrm{hpf}$. The larvae were treated with $10 \mu \mathrm{M}$ PD168393 and $3 \mu \mathrm{M}$ SIS3 from $84 \mathrm{hpf}$ to $120 \mathrm{hpf}$. All inhibitors were dissolved in DMSO and added to egg water. Control fish were incubated in $1 \%$ DMSO in egg water.

Statistical analysis. No statistical methods were used to predetermine sample size. The experiments were not randomized. The investigators were not blinded to allocation during experiments or outcome assessment, except for the data shown in Fig. 4e-s. GraphPad software was used to perform statistical analysis. Data are represented as mean \pm s.e.m. $P$-values were calculated by two-tailed Student's $t$ test.

Data availability. The authors declare that all data supporting the findings of this study are available within the article and its Supplementary Information files or from the corresponding author upon reasonable request. Microarray raw and normalized data have been deposited in the NCBI-GEO database under the accession code: GSE89259.
Received: 10 March 2017 Accepted: 27 October 2017

Published online: 01 December 2017

\section{References}

1. Steinhauser, M. L. \& Lee, R. T. Regeneration of the heart. EMBO Mol. Med. 3, 701-712 (2011).

2. Mozaffarian, D. et al. Heart Disease and Stroke Statistics-2016 Update: a report from the American Heart Association. Circulation 133, e38-e360 (2016).

3. Chong, J. J. et al. Human embryonic-stem-cell-derived cardiomyocytes regenerate non-human primate hearts. Nature 510, 273-277 (2014).

4. Bersell, K., Arab, S., Haring, B. \& Kühn, B. Neuregulin1/ErbB4 signaling induces cardiomyocyte proliferation and repair of heart injury. Cell 138, 257-270 (2009).

5. Qian, L. et al. In vivo reprogramming of murine cardiac fibroblasts into induced cardiomyocytes. Nature 485, 593-598 (2012).

6. Poss, K. D., Wilson, L. G. \& Keating, M. T. Heart regeneration in zebrafish. Science 298, 2188-2190 (2002).

7. Chablais, F., Veit, J., Rainer, G. \& Jaźwińska, A. The zebrafish heart regenerates after cryoinjury-induced myocardial infarction. BMC Dev. Biol. 11, 21 (2011)

8. Wang, J. et al. The regenerative capacity of zebrafish reverses cardiac failure caused by genetic cardiomyocyte depletion. Development 138, 3421-3430 (2011).

9. Jopling, C. et al. Zebrafish heart regeneration occurs by cardiomyocyte dedifferentiation and proliferation. Nature 464, 606-609 (2010).

10. Kikuchi, K. et al. Primary contribution to zebrafish heart regeneration by gata 4 (+) cardiomyocytes. Nature 464, 601-605 (2010).

11. Wu, C.-C. C. et al. Spatially resolved genome-wide transcriptional profiling identifies BMP signaling as essential regulator of zebrafish cardiomyocyte regeneration. Dev. Cell 36, 36-49 (2016).

12. Kikuchi, K. Advances in understanding the mechanism of zebrafish heart regeneration. Stem Cell Res. 13, 542-555 (2014).

13. Marín-Juez, R. et al. Fast revascularization of the injured area is essential to support zebrafish heart regeneration. Proc. Natl Acad. Sci. USA 113, 11237-11242 (2016).

14. Gemberling, M., Karra, R., Dickson, A. L. \& Poss, K. D. Nrg1 is an injuryinduced cardiomyocyte mitogen for the endogenous heart regeneration program in zebrafish. Elife 1, 4 (2015).

15. D'Uva, G. et al. ERBB2 triggers mammalian heart regeneration by promoting cardiomyocyte dedifferentiation and proliferation. Nat. Cell Biol. 17, 627-638 (2015).

16. Liu, J. et al. A dual role for ErbB2 signaling in cardiac trabeculation. Development 137, 3867-3875 (2010).

17. Rasouli, S. J. \& Stainier, D. Y. Regulation of cardiomyocyte behavior in zebrafish trabeculation by Neuregulin 2a signaling. Nat. Commun. 8, 15281 (2017).

18. Polizzotti, B. D. et al. Neuregulin stimulation of cardiomyocyte regeneration in mice and human myocardium reveals a therapeutic window. Sci. Transl. Med. 7, $281 \mathrm{ra} 45$ (2015).

19. Ahuja, S., Dogra, D., Stainier, D. Y. \& Reischauer, S. Id4 functions downstream of Bmp signaling to restrict TCF function in endocardial cells during atrioventricular valve development. Dev. Biol. 412, 71-82 (2016).

20. Siegel, P. M. \& Massagué, J. Cytostatic and apoptotic actions of TGF-beta in homeostasis and cancer. Nat. Rev. Cancer 3, 807-821 (2003).

21. Massagué, J. \& Gomis, R. R. The logic of TGFbeta signaling. FEBS Lett. 580, 2811-2820 (2006).

22. Massagué, J. TGF $\beta$ signalling in context. Nat. Rev. Mol. Cell Biol. 13, 616-630 (2012).

23. Sartori, R., Gregorevic, P. \& Sandri, M. TGF $\beta$ and BMP signaling in skeletal muscle: potential significance for muscle-related disease. Trends Endocrinol. Metab. 25, 464-471 (2014).

24. Derynck, R. \& Zhang, Y. E. Smad-dependent and Smad-independent pathways in TGF-beta family signalling. Nature 425, 577-584 (2003).

25. Castillero, E. et al. Cardiac myostatin upregulation occurs immediately after myocardial ischemia and is involved in skeletal muscle activation of atrophy. Biochem. Biophys. Res. Commun. 457, 106-111 (2015).

26. Yndestad, A. et al. Elevated levels of activin A in heart failure: potential role in myocardial remodeling. Circulation 109, 1379-1385 (2004).

27. Li, R. K. et al. Overexpression of transforming growth factor-betal and insulinlike growth factor-I in patients with idiopathic hypertrophic cardiomyopathy. Circulation 96, 874-881 (1997).

28. Euler-Taimor, G. \& Heger, J. The complex pattern of SMAD signaling in the cardiovascular system. Cardiovasc. Res. 69, 15-25 (2006).

29. Zeisberg, E. M. et al. Endothelial-to-mesenchymal transition contributes to cardiac fibrosis. Nat. Med. 13, 952-961 (2007). 
30. Koitabashi, N. et al. Pivotal role of cardiomyocyte TGF- $\beta$ signaling in the murine pathological response to sustained pressure overload. J. Clin. Invest. 121, 2301-2312 (2011).

31. Chablais, F. \& Jazwinska, A. The regenerative capacity of the zebrafish heart is dependent on TGF $\beta$ signaling. Development 139, 1921-1930 (2012).

32. Whittemore, L.-A. A. et al. Inhibition of myostatin in adult mice increases skeletal muscle mass and strength. Biochem. Biophys. Res. Commun. 300, 965-971 (2003).

33. Haidet, A. M. et al. Long-term enhancement of skeletal muscle mass and strength by single gene administration of myostatin inhibitors. Proc. Natl Acad. Sci. USA 105, 4318-4322 (2008).

34. Biesemann, N. et al. Myostatin regulates energy homeostasis in the heart and prevents heart failure. Circ. Res. 115, 296-310 (2014).

35. Rodgers, B. D. et al. Myostatin represses physiological hypertrophy of the heart and excitation-contraction coupling. J. Physiol. 587, 4873-4886 (2009)

36. Wagner, K. R., Liu, X., Chang, X. \& Allen, R. E. Muscle regeneration in the prolonged absence of myostatin. Proc. Natl Acad. Sci. USA 102, 2519-2524 (2005).

37. Latres, E. et al. Myostatin blockade with a fully human monoclonal antibody induces muscle hypertrophy and reverses muscle atrophy in young and aged mice. Skelet Muscle 5, 34 (2015).

38. Jaźwińska, A., Badakov, R. \& Keating, M. T. Activin-betaA signaling is required for zebrafish fin regeneration. Curr. Biol. 17, 1390-1395 (2007).

39. Biesemann, N. et al. Myostatin induces interstitial fibrosis in the heart via TAK1 and p38. Cell Tissue Res. 361, 779-787 (2015).

40. Sallin, P., Preux Charles, A.-S. S., de, Duruz, V., Pfefferli, C. \& Jaźwińska, A. A dual epimorphic and compensatory mode of heart regeneration in zebrafish. Dev. Biol. 399, 27-40 (2015)

41. Rebbapragada, A., Benchabane, H., Wrana, J. L., Celeste, A. J. \& Attisano, L. Myostatin signals through a transforming growth factor beta-like signaling pathway to block adipogenesis. Mol. Cell Biol. 23, 7230-7242 (2003).

42. Chen, X., Weisberg, E., Fridmacher, V. \& Watanabe, M. Smad4 and FAST-1 in the assembly of activin-responsive factor. Nature 389, 85-89 (1997).

43. Chen, X., Rubock, M. J. \& Whitman, M. A transcriptional partner for MAD proteins in TGF-beta signalling. Nature 383, 691-696 (1996).

44. Labbé, E., Silvestri, C., Hoodless, P. A., Wrana, J. L. \& Attisano, L. Smad2 and Smad3 positively and negatively regulate TGF beta-dependent transcription through the forkhead DNA-binding protein FAST2. Mol. Cell 2, 109-120 (1998).

45. Jonk, L., Itoh, S., Heldin, C.-H., Dijke, Pten. \& Kruijer, W. Identification and functional characterization of a smad binding element (SBE) in the JunB promoter that acts as a transforming growth factor- $\beta$, activin, and bone morphogenetic protein-inducible Enhancer. J. Biol. Chem. 273, 21145-21152 (1998).

46. Stroschein, S. L., Wang, W. \& Luo, K. Cooperative binding of Smad proteins to two adjacent DNA elements in the plasminogen activator inhibitor-1 promoter mediates transforming growth factor beta-induced smad-dependent transcriptional activation. J. Biol. Chem. 274, 9431-9441 (1999).

47. Boxtel, A. Lvan. et al. A temporal window for signal activation dictates the dimensions of a nodal signaling domain. Dev. Cell 35, 175-185 (2015).

48. Casari, A. et al. A Smad3 transgenic reporter reveals TGF-beta control of zebrafish spinal cord development. Dev. Biol. 396, 81-93 (2014).

49. Choi, W.-Y. Y. et al. In vivo monitoring of cardiomyocyte proliferation to identify chemical modifiers of heart regeneration. Development 140, 660-666 (2013).

50. Jinnin, M., Ihn, H. \& Tamaki, K. Characterization of SIS3, a novel specific inhibitor of Smad3, and its effect on transforming growth factor-betal-induced extracellular matrix expression. Mol. Pharmacol. 69, 597-607 (2006).

51. Denis, J.-F. F. et al. Activation of Smad2 but not Smad3 is required to mediate TGF- $\beta$ signaling during axolotl limb regeneration. Development 143, 3481-3490 (2016).

52. Inman, G. J. et al. SB-431542 is a potent and specific inhibitor of transforming growth factor-beta superfamily type I activin receptor-like kinase (ALK) receptors ALK4, ALK5, and ALK7. Mol. Pharmacol. 62, 65-74 (2002).

53. Lee, S. J. \& McPherron, A. C. Regulation of myostatin activity and muscle growth. Proc. Natl Acad. Sci. USA 98, 9306-9311 (2001)

54. Ho, D. M. \& Whitman, M. TGF-beta signaling is required for multiple processes during Xenopus tail regeneration. Dev. Biol. 315, 203-216 (2008).

55. Thenappan, A. et al. Role of transforming growth factor beta signaling and expansion of progenitor cells in regenerating liver. Hepatology 51, 1373-1382 (2010)

56. Chen, H. et al. Analysis of ventricular hypertrabeculation and noncompaction using genetically engineered mouse models. Pediatr. Cardiol. 30, 626-634 (2009).

57. Wang, T. et al. The immunophilin FKBP12 functions as a common inhibitor of the TGF beta family type I receptors. Cell 86, 435-444 (1996).

58. Kretzschmar, M., Doody, J., Timokhina, I. \& Massagué, J. A mechanism of repression of TGFbeta/Smad signaling by oncogenic Ras. Genes Dev. 13, 804-816 (1999).

59. Zhou, P. \& Pu, W. T. Recounting cardiac cellular composition. Circ. Res. 118, 368-370 (2016).
60. Brown, K. A., Pietenpol, J. A. \& Moses, H. L. A tale of two proteins: differential roles and regulation of Smad2 and Smad3 in TGF-beta signaling. J. Cell Biochem. 101, 9-33 (2007).

61. Petersen, M. et al. Smad2 and Smad3 have opposing roles in breast cancer bone metastasis by differentially affecting tumor angiogenesis. Oncogene 29, 1351-1361 (2010).

62. Míguez, D. G., Gil-Guiñón, E., Pons, S. \& Martí, E. Smad2 and Smad3 cooperate and antagonize simultaneously in vertebrate neurogenesis. J. Cell Sci. 126, 5335-5343 (2013).

63. Lyons, D. A. et al. erbb3 and erbb2 are essential for schwann cell migration and myelination in zebrafish. Curr. Biol. 15, 513-524 (2005).

64. Takeuchi, J. K. et al. Chromatin remodelling complex dosage modulates transcription factor function in heart development. Nat. Commun. 2, 187 (2011).

65. Burns, G. C. \& MacRae, C. A. Purification of hearts from zebrafish embryos. Biotechniques 40, 274 (2006).

66. Cermak, T. et al. Efficient design and assembly of custom TALEN and other TAL effector-based constructs for DNA targeting. Nucleic Acids Res. 39, e82 (2011).

67. Chipuk, J. E. et al. The androgen receptor represses transforming growth factorbeta signaling through interaction with Smad3. J. Biol. Chem. 277, 1240-1248 (2002).

68. Bae, Y.-K. K. et al. Anatomy of zebrafish cerebellum and screen for mutations affecting its development. Dev. Biol. 330, 406-426 (2009).

69. Beis, D. et al. Genetic and cellular analyses of zebrafish atrioventricular cushion and valve development. Development 132, 4193-4204 (2005).

70. Reischauer, S., Arnaout, R., Ramadass, R. \& Stainier, D. Y. Actin binding GFP allows $4 \mathrm{D}$ in vivo imaging of myofilament dynamics in the zebrafish heart and the identification of Erbb2 signaling as a remodeling factor of myofibril architecture. Circ. Res. 115, 845-856 (2014).

\section{Acknowledgements}

We would like to thank Veronica Uribe Sokolov, Anoop V. Cherian, Vanesa Jiménez Amilburu, Michele Marass, Jason Kuan Han Lai, and Raoul F.V. Germano for helpful discussions, Chi-Chung Wu, Rubén Marín-Juez, Arica Beisaw, and Alethia Villasenor for comments on the manuscript, Srinivas Allanki for help with statistics, Radhan Ramadas for microscopy guidance, Beate Grohmann for help with cryosectioning, Anabela Bensimon Brito for help with LMD, Hans-Martin Maischein for help with injections, and Rita Retzloff and Martin Laszczyk for zebrafish care. This work was supported by fellowships from the IMPRS-HLR (D.D.), the DFG RE3148/1-1 and SFB 1213, project B1 (S.R.) and grants from the NIH (HL54737), the Packard Foundation, the Leduce Foundation and the Max Planck Society (D.Y.R.S.).

\section{Author contributions}

D.D. designed the study, performed the experiments, analyzed the data and wrote the manuscript; S.A. performed parts of the histology experiments; H.-T.K. performed in situ hybridization experiments; S.J.R. provided an unpublished reagent; D.Y.R.S. and S.R. designed the study, analyzed the data, wrote the manuscript and supervised the work. All the authors commented on the manuscript.

\section{Additional information}

Supplementary Information accompanies this paper at doi:10.1038/s41467-017-01950-1.

Competing interests: The authors declare no competing financial interests.

Reprints and permission information is available online at http://npg.nature.com/ reprintsandpermissions/

Publisher's note: Springer Nature remains neutral with regard to jurisdictional claims in published maps and institutional affiliations.

Open Access This article is licensed under a Creative Commons Attribution 4.0 International License, which permits use, sharing, adaptation, distribution and reproduction in any medium or format, as long as you give appropriate credit to the original author(s) and the source, provide a link to the Creative Commons license, and indicate if changes were made. The images or other third party material in this article are included in the article's Creative Commons license, unless indicated otherwise in a credit line to the material. If material is not included in the article's Creative Commons license and your intended use is not permitted by statutory regulation or exceeds the permitted use, you will need to obtain permission directly from the copyright holder. To view a copy of this license, visit http://creativecommons.org/ licenses/by/4.0/

(C) The Author(s) 2017 